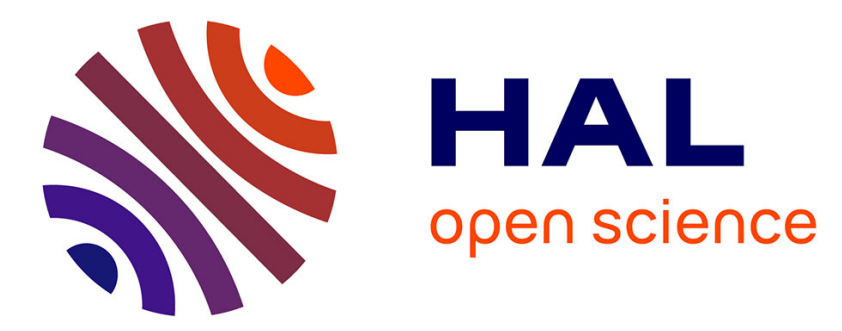

\title{
Existence and robustness of phase-locking in coupled Kuramoto oscillators under mean-field feedback
}

\author{
Alessio Franci, Antoine Chaillet, William Pasillas-Lépine
}

\section{To cite this version:}

Alessio Franci, Antoine Chaillet, William Pasillas-Lépine. Existence and robustness of phase-locking in coupled Kuramoto oscillators under mean-field feedback. Automatica, 2011, 47, pp.1193-1202. 10.1016/j.automatica.2011.03.003 . hal-00526066

\section{HAL Id: hal-00526066 https://hal.science/hal-00526066}

Submitted on 17 Jun 2011

HAL is a multi-disciplinary open access archive for the deposit and dissemination of scientific research documents, whether they are published or not. The documents may come from teaching and research institutions in France or abroad, or from public or private research centers.
L'archive ouverte pluridisciplinaire HAL, est destinée au dépôt et à la diffusion de documents scientifiques de niveau recherche, publiés ou non, émanant des établissements d'enseignement et de recherche français ou étrangers, des laboratoires publics ou privés. 


\title{
Existence and robustness of phase-locking in coupled Kuramoto oscillators under mean-field feedback
}

\author{
Alessio Franci ${ }^{\mathrm{a}}$, Antoine Chaillet ${ }^{\mathrm{b}}$, William Pasillas-Lépine ${ }^{\mathrm{c}}$ \\ ${ }^{a}$ Univ. Paris Sud 11 - L2S - Supélec, 3, rue Joliot-Curie, 91192 Gif-sur-Yvette, France. \\ ${ }^{\mathrm{b}}$ EECI - L2S - Univ. Paris Sud 11 - Supélec, same address. \\ ${ }^{\mathrm{c}}$ CNRS-L2S, same address.
}

\begin{abstract}
Motivated by the recent development of Deep Brain Stimulation (DBS) for neurological diseases, we study a network of interconnected oscillators under the influence of mean-field feedback and analyze the robustness of its phase-locking with respect to general inputs. Under standard assumptions, this system can be reduced to a modified version of the Kuramoto model of coupled nonlinear oscillators. In the first part of the paper we present an analytical study on the existence of phase-locked solutions under generic interconnection and feedback configurations. In particular we show that, in general, no oscillating phaselocked solutions can co-exist with any non-zero proportional mean-field feedback. In the second part we prove some robustness properties of phase-locked solutions (namely total stability). This general result allows in particular to justify the persistence of practically phase-locked states if sufficiently small feedback gains are applied, and to give explicit necessary conditions on the intensity of a desynchronizing mean-field feedback. Furthermore, the Lyapunov function used in the analysis provides a new characterization of the robust phase-locked configurations in the Kuramoto system with symmetric interconnections.
\end{abstract}

Key words: Phase-locking, Mean-field feedback, Kuramoto oscillators, Deep Brain Stimulation

\section{Introduction}

In most automatic control applications, synchronization is a goal to achieve: for instance, formations of autonomous vehicles (Sarlette, 2009; Sepulchre et al., 2007, 2008), consensus protocols (Scardovi et al., 2007; Olfati-Saber and Murray, 2004; Sarlette, 2009) and master-slave control of mechanical systems (Pavlov et al., 2006) can all be formulated as a synchronization objective. For some applications, however, synchronization is an undesired effect and the aim of the control law is then to "desynchronize". One of these applications is that of Deep Brain Stimulation for Parkinson's Disease (PD), which is the motivation of the present article. Under healthy conditions subthalamic nucleus (STN) neurons fire in an uncorrelated (i.e., desynchronized)

Email addresses: Alessio.Franci@lss.supelec.fr (Alessio Franci), Antoine.Chaillet@supelec.fr (Antoine Chaillet), William.Pasillas-Lepine@lss.supelec.fr (William Pasillas-Lépine).

1 This work was financially supported by the European Comission through the FP7 NoE HYCON2, and by the French CNRS through the PEPS project TREMBATIC. manner (Nini et al., 1995; Sarma et al., 2010). In PD patients, STN neurons form a cluster of synchronous periodic activity that leads to limb tremor (Volkmann et al., 1996).

While the exact physiological mechanisms that leads to this phenomenon are still unclear, experimental evidences suggest that low frequency oscillations (beta range, $10-30 \mathrm{~Hz}$ ) serve as a trigger for high frequency synchronous bursting correlated with movement disorders (Sarma et al., 2010; Lopez-Azcarate et al., 2010; Plenz and Kital, 1999; Rosa et al., 2010). In order to overcome tolerance to pharmaceutical therapies, many patients undergo Deep Brain Stimulation (DBS). Through a pair of implanted electrodes, a low voltage "high"-frequency (>100 Hz) electrical input is permanently injected in the STN. This leads to a drastic reduction of the physical symptoms (Benabid et al., 1991).

At present this electrical signal is periodic and generated by a standard artificial pacemaker (open-loop control) and is consequently not optimized for the purpose. Despite its therapeutic success, little is still known 
about the exact functioning of DBS (Hammond et al., 2008). For each patient an empirical parameter tuning is needed, which may take up to several days and which is not guaranteed to be effective (Rodriguez-Oroz and et al., 2005). Moreover patients can develop side effects or tolerance to DBS (Kumar et al., 2003) with long term treatment. Also, the permanent electrical stimulation leads to a fast discharge of the pacemaker batteries and, consequently, to further surgical operations to change them.

In order to both provide theoretical justifications to DBS and to bypass the above limitations by exploiting cerebral measurements, we develop a rigorous analysis based on a simplified model. More precisely, we analyze synchronization and desynchronization phenomena in coupled complex Landau-Stuart oscillators subject to a scalar input modeling the effect of DBS. The DBS signal is taken proportional to the mean-field of the neuronal population. Due to heterogeneities in the medium, the contribution of each neuron to the mean-field is seen as an unknown parameter. In the same way, the influence of the DBS signal on each neuron is modeled as an unknown gain. The coupling topology is also taken to be arbitrary, allowing for a general time-invariant synaptic interconnection. This approach thus allows to represent any recording-stimulation setup as well as any coupling topology. Nonetheless, we point out that it does not model details of the neuronal dynamics, nor the electrode setup. Under standard assumptions, our model reduces to a modified version of Kuramoto coupled oscillators. This model, originally developed in the seminal works (Kuramoto, 1984; Winfree, 1980), has been already exploited to analyze both synchronization and desynchronization phenomena. In particular the robustness properties of phase-locked solutions to exogenous inputs has been partially addressed in the infinite dimensional case (Pyragas et al., 2008; Daniels, 2005; Acebrón et al., 2005; Kuramoto, 1984), while in the finite case it has only been studied either through simulations (Brown et al., 2003; Cumin and Unsworth, 2007; Maistrenko et al., 2005), or for constant inputs (Chopra and Spong, 2009; Van Hemmen and Wreszinski, 1993; Jadbabaie et al., 2004; Dörfler and Bullo, 2011). Only recently the interest of the scientific community has focused on desynchronization, in particular in relation with neurological pathologies (Maistrenko et al., 2005; Pyragas et al., 2008; Tukhlina et al., 2007; Tass, 2003). In Section 2, we derive an original model of interconnected oscillators under mean-field feedback. In Section 3, after having formally defined the concept of phase-locked solutions, we characterize them through a generalized fixed-point equation and we show that, for a generic class of interconnections between the oscillators, the existence of perfectly phase-locked oscillating solutions is not compatible with any non-zero meanfield proportional feedback. This analytical result, illustrated through simulations, confirms the expectations of a closed-loop desynchronizing strategy. Moreover, the evidence of "practical" phase-locking for small feedback gains observed in simulations, along with the aim of computing necessary conditions on the feedback gains which would assure effective desynchronization, leads us to a robustness analysis of phase-locked solutions with respect to general time-varying inputs (cf. Section 4). In particular, we prove local input-to-state stability of the phase-locked states with respect to small inputs (total stability) for general bidirectional interconnection topologies. The use of an explicit Lyapunov function allows to give explicit bounds on the size of the tolerated inputs for some particular interconnection topologies. To the best of our knowledge this is the first attempt to analyze the robustness of phase-locking to time-varying inputs in the finite dimensional Kuramoto system with symmetric interconnection topology. In practice, these bounds, together with an approximate knowledge of the interconnection topology between the oscillators and their natural frequencies' distribution, can be used to compute the necessary minimum value of the intensity of mean-field feedback to desynchronize the oscillators. We evoke future work in Section 5. The proofs of the main results are given in Section 6.

Notations. For a set $A \subset \mathbb{R}$, and $a \in A, A_{>a}$ denotes the set $\{x \in A: x \geq a\} .|x|_{2}$ denotes the $E u$ clidean norm of $x \in \mathbb{R}^{n}$ and $|x|_{\infty}$ denotes its infinity norm. When clear from the context, we simply denote the Euclidean norm as $|x|$. For $\mathcal{A} \subset \mathbb{R}^{n}$ and $x \in \mathbb{R}^{n}$, $|x|_{\mathcal{A}}:=\inf _{y \in \mathcal{A}}|y-x| \cdot \mathcal{B}(x, R)$ refers to the closed ball of radius $R$ centered at $x$ in the Euclidean norm, i.e. $\mathcal{B}(x, R):=\left\{z \in \mathbb{R}^{n}:|x-z| \leq R\right\} . \mathrm{T}^{n}$ is the $n$-Torus. $\nabla_{x}$ is the gradient vector with respect to $x$, i.e. $\nabla_{x}=$ $\left(\frac{\partial}{\partial x_{1}}, \ldots, \frac{\partial}{\partial x_{n}}\right)$. Given $x \in \mathbb{R}^{n}$ and $a \in \mathbb{R},(x \bmod a):=$ $\left[x_{i} \bmod a\right]_{i=1, \ldots, n}$, where $\bmod$ denotes the modulo operator. Given a function $f: \mathbb{R}^{n} \rightarrow \mathbb{R},\left.f\right|_{\mathcal{A}}: \mathcal{A} \rightarrow \mathbb{R}$ denotes its restriction to $\mathcal{A} \subset \mathbb{R}^{n}$, that is $\left.f\right|_{\mathcal{A}}(x):=f(x)$ for all $x \in \mathcal{A}$. If $u: \mathbb{R}_{\geq 0} \rightarrow \mathbb{R}^{n}$ denotes a measurable signal, locally essentially bounded, $\|u\|:=\operatorname{ess} \sup _{t>0}|u(t)|$. A continuous function $\alpha: \mathbb{R}_{>0} \rightarrow \mathbb{R}_{>0}$ is said to be of class $\mathcal{K}$ if it is increasing and $\alpha(0)=0$. It is said to be of class $\mathcal{K}_{\infty}$ if it is of class $\mathcal{K}$ and $\alpha(s) \rightarrow \infty$ as $s \rightarrow \infty$. A function $\beta: \mathbb{R}_{>0} \times \mathbb{R}_{>0} \rightarrow \mathbb{R}_{>0}$ is said to be of class $\mathcal{K} \mathcal{L}$ if $\beta(\cdot, t) \in \mathcal{K}$ for any fixed $t \geq 0$ and $\beta(s, \cdot)$ is continuous non-increasing and tends to zero at infinity for any fixed $s \geq 0$. $\mu^{n}$ is the Lebesgue measure on $\mathbb{R}^{n}$, and for almost all ( $\forall$ a.a.) denotes the equivalence operation with respect to this measure. The vector with all unitary components in $\mathbb{R}^{n}$ is denoted by $\overrightarrow{1}_{n}$.

\section{Model Derivation}

Although biophysically substantiated models of neurons, such as (Hodgkin and Huxley, 1952), provide a detailed and accurate description of the membrane voltage dynamics, their complexity and the large number of involved variables and parameters hamper the mathematical treatment of the model and the appreciation of 
the mechanisms underlying the observed phenomena. In contrast, simple phenomenological models, although less precise, often allow for a comprehension of the basic dynamical mechanisms, which can then be generalized for a broader class of models. Neurons with periodic internal dynamics, that are those of interest in the present analysis, show a two-dimensional limit cycle in the space of membrane voltage and ion concentration that can arise from different types of bifurcation (cf.e.g. (Izhikevich, 2007, Sections 6.1.3,6.1.4)). A simple representation of this limit cycle is given by the Landau-Stuart oscillator (Kuramoto, 1984):

$$
\dot{z}=\left(\mathrm{i} \omega_{\circ}+\rho^{2}-|z|^{2}\right) z, \quad z \in \mathbb{C},
$$

which represents a normal form of the Andronov-Hopf bifurcation, where $\omega_{\circ} \in \mathbb{R}$ and $\rho \in \mathbb{R}_{>0}$ denote the natural frequency and the radius of the oscillation, respectively. While the coupling between real neurons can rely on different physical mechanisms (electrical diffusive coupling or gap-junction, impulsive coupling, synaptic noise coupling, etc.), we assume diffusive coupling between the oscillators, in order to derive a mathematically treatable model. The same approach has been exploited (Maistrenko et al., 2005; Pyragas et al., 2008; Tukhlina et al., 2007; Tass, 2003). The model for $N \in \mathbb{N}_{>1}$ coupled oscillators is then given by

$\dot{z}_{i}=\left(\mathrm{i} \omega_{i}+\rho_{i}^{2}-\left|z_{i}\right|^{2}\right) z_{i}+\sum_{j=1}^{N} \kappa_{i j}\left(z_{j}-z_{i}\right), \quad \forall i=1, \ldots, N$

where $\kappa_{i j}, i, j=1, \ldots, N$, denotes the coupling gain from oscillator $j$ to oscillator $i$. We denote $\omega:=\left[\omega_{i}\right]_{i=1, \ldots, N} \in \mathbb{R}^{N}$ as the vector of natural frequencies. As in practice the neuronal interconnection is poorly known, we allow $\kappa_{i j}, i, j=1, \ldots, N$, to be arbitrary in our study. The possibility of considering any interconnection topology is an interesting particularity of the approach presented here. Furthermore, the presence of a limited number of electrodes and their large size with respect to the neuronal scale, makes the mean-field (i.e. the mean neurons membrane voltages) the only realistic measurement for DBS. In the same way, the unknown distances from the neurons to the electrodes and the unknown conductivity of nearby tissues make the contribution of each neuron to the overall recording both heterogeneous and unknown. Consequently the only measurement assumed to be available for DBS is the weighted sum of the neuron membrane voltages. The output of our system is therefore

$$
y:=\sum_{j=1}^{N} \alpha_{j} \operatorname{Re}\left(z_{j}\right)
$$

which is referred to as the mean-field of the ensemble, where $\alpha:=\left[\alpha_{j}\right]_{j=1, \ldots, N} \in \mathbb{R}_{\geq 0}^{N}$ describes the influence of each neuron on the electrode's recording. Similarly, we define $\beta:=\left[\beta_{j}\right]_{j=1, \ldots, N} \in \mathbb{R}^{N}$, as the gain of the electrical input on each neuron. It is assumed to be unknown. The pair $(\alpha, \beta)$ thus defines the stimulationregistration setup. The dynamics of $N$ coupled oscillators under mean-field feedback then reads:

$\dot{z}_{i}=\left(\mathrm{i} \omega_{i}+\rho_{i}^{2}-\left|z_{i}\right|^{2}\right) z_{i}+\sum_{j=1}^{N} \kappa_{i j}\left(z_{j}-z_{i}\right)+\beta_{i} \sum_{j=1}^{N} \alpha_{j} \operatorname{Re}\left(z_{j}\right)$,

for all $i=1, \ldots, N$. Let us briefly compare the above model to existing ones. In (Rosenblum and Pikovsky, 2004; Tukhlina et al., 2007) the global dynamics of the network is modeled as a single Landau-Stuart oscillator, exploiting the fact that oscillators are synchronized. Hence that model is valid only near the synchronous state. On the contrary (3) is valid for both synchronized and desynchronized behaviors. In (Popovych et al., 2006) the authors use a population approach with all-toall coupling that makes the results valid only for large number of oscillators. Our paper allows for general couplings and number of agents. Finally, we consider a real output as opposed to the complex output assumed in (Popovych et al., 2006).

In order to simplify the analysis, we make the assumption that each oscillator evolves with constant radius.

Assumption 1 (Constant radii) For all $i=1, \ldots, N$ there exists a constant $r_{i}>0$ such that the solution of (3) satisfies $\left|z_{i}(t)\right|=r_{i}$, for all $t \geq 0$.

This assumption is commonly made in synchronization studies (Acebrón et al., 2005; Aeyels and Rogge, 2004; Jadbabaie et al., 2004; Van Hemmen and Wreszinski, 1993; Brown et al., 2003; Kuramoto, 1984), and is justified by the normal hyperbolicity of the stable limit cycle of (1) that let the oscillation persist under external perturbations (cf. e.g. (Hoppensteadt and Izhikevich, 1997, Chapter 4.3)).

Letting $z_{i}=r_{i} e^{\mathrm{i} \theta_{i}}$, which defines the phase $\theta_{i} \in \mathrm{T}^{1}$ of each oscillator, we get from Assumption 1 that $\dot{z}_{i}=$ $\dot{r}_{i} e^{\mathrm{i} \theta_{i}}+\mathrm{i} r_{i} \dot{\theta}_{i} e^{\mathrm{i} \theta_{i}}=\mathrm{i} r_{i} \dot{\theta}_{i} e^{\mathrm{i} \theta_{i}}$. Dividing each side of this equation by $r_{i} e^{\mathrm{i} \theta_{i}}$, and extracting the imaginary part of both sides, we get from (3) that

$\dot{\theta}_{i}=\omega_{i}+\sum_{j=1}^{N} \kappa_{i j} \frac{r_{j}}{r_{i}} \sin \left(\theta_{j}-\theta_{i}\right)-\beta_{i} \sin \left(\theta_{i}\right) \sum_{j=1}^{N} \alpha_{j} \frac{r_{j}}{r_{i}} \cos \left(\theta_{j}\right)$.

We can now use the trigonometric identity $\sin \theta_{i} \cos \theta_{j}=$ $\frac{1}{2} \sin \left(\theta_{j}+\theta_{i}\right)-\frac{1}{2} \sin \left(\theta_{j}-\theta_{i}\right)$ to derive

$\dot{\theta}_{i}=\omega_{i}+\sum_{j=1}^{N}\left(k_{i j}+\gamma_{i j}\right) \sin \left(\theta_{j}-\theta_{i}\right)-\sum_{j=1}^{N} \gamma_{i j} \sin \left(\theta_{j}+\theta_{i}\right)$, 
for all $i=1, \ldots, N$, where

$$
k=\left[k_{i j}\right]_{i, j=1, \ldots, N}:=\left[\kappa_{i j} \frac{r_{j}}{r_{i}}\right]_{i, j=1, \ldots, N} \in \mathbb{R}^{N \times N}
$$

is referred to as the coupling matrix, and

$$
\gamma=\left[\gamma_{i j}\right]_{i, j=1, \ldots, N}:=\left[\frac{\beta_{i}}{2} \frac{\alpha_{j} r_{j}}{r_{i}}\right]_{i, j=1, \ldots, N} \in \mathbb{R}^{N \times N}
$$

defines the feedback gain. We also define the modified coupling matrix, $\Gamma \in \mathbb{R}^{N \times N}$, as

$$
\Gamma:=\left[\Gamma_{i j}\right]_{i, j=1, \ldots, N}=\left[k_{i j}+\gamma_{i j}\right]_{i, j=1, \ldots, N} .
$$

Our study is based on the incremental dynamics of (4), defined, for all $i, j=1, \ldots, N$, by

$$
\begin{aligned}
& \dot{\theta}_{i}-\dot{\theta}_{j}=\omega_{i}-\omega_{j}-\sum_{n=1}^{N}\left(\gamma_{i n} \sin \left(\theta_{j}+\theta_{i}\right)+\gamma_{j n} \sin \left(\theta_{n}+\theta_{i}\right)\right)+ \\
& \sum_{n=1}^{N}\left(\Gamma_{i n} \sin \left(\theta_{n}-\theta_{i}\right)-\Gamma_{j n} \sin \left(\theta_{n}-\theta_{j}\right)\right)
\end{aligned}
$$

The model (4) appears to be new in the literature and allows, by properly choosing $\alpha, \beta$ and $\kappa$, to encompass all kinds of interconnection topologies and recordingstimulation setups. We stress that the use of a nonzero feedback gains $\gamma$ breaks the $T^{1}$ (i.e global phase shift (Sepulchre et al., 2007, Eq. (8))) symmetry of the original Kuramoto system. This complicates the analysis, but allows for new desynchronization expectations.

\section{Phase-locked solutions}

\subsection{Definitions}

We start this section by formally defining the concept of phase-locking. Roughly speaking, a phase-locked solution can be interpreted as a fixed point of the incremental dynamics (8). We distinguish solutions that exhibit collective oscillations (pathological case for DBS) from non-oscillating ones (neuronal inhibition).

Definition 1 (Phase-locked solution) A solution $\left\{\theta_{i}^{*}\right\}_{i=1, \ldots, N}$ of (4) is said to be phase-locked if it satisfies

$$
\dot{\theta}_{j}^{*}(t)-\dot{\theta}_{i}^{*}(t)=0, \quad \forall i, j=1, \ldots, N, \forall t \geq 0 .
$$

A phase-locked solution is oscillating if, in addition, $\dot{\theta}_{i}^{*}(t) \neq 0$, for almost all $t \geq 0$ and all $i=1, \ldots, N$.

In other words, for oscillating phase-locked solutions, the discharge rhythm is the same for each neuron, which corresponds to a synchronous (pathological) activity, while in the non oscillating case the neurons are in a quiescent non pathological state. The above definition of phase-locking corresponds to that of "Frequency (Huygens) Synchronization" (cf. e.g (Fradkov, 2007)). It is trivially equivalent to the existence of a matrix $\Delta:=$ $\left[\Delta_{i j}\right]_{i, j=1, \ldots, N}$, such that

$$
\theta_{j}^{*}(t)-\theta_{i}^{*}(t)=\Delta_{i j}, \quad \forall i, j=1, \ldots, N, \forall t \geq 0,
$$

or to the existence of a measurable function $\Omega: \mathbb{R}_{\geq 0} \rightarrow \mathbb{R}$ such that, for each $i=1, \ldots, N$,

$$
\theta_{i}^{*}(t)=\int_{0}^{t} \Omega(s) d s+\theta_{i}^{*}(0), \quad \forall t \geq 0
$$

where $\Omega$ is the instantaneous collective frequency of oscillation, that is $\dot{\theta}_{i}^{*}(t)=\Omega(t)$ for all $i=1, \ldots, N$. In case of oscillating phase-locking, $\Omega(t) \neq 0$ for almost all $t \geq 0$.

In the Kuramoto system without mean-field feedback, the oscillating and non-oscillating cases are equivalent due to the $T^{1}$ symmetry, which guarantees invariance to a common phase drift such as a nonzero mean natural frequency (Sepulchre et al., 2007, Eq. (8)). The presence of the mean-field feedback, on the contrary, breaks this symmetry, leading to new interesting phenomena, such as oscillator "death" and mean-field approach to desynchronization (Franci et al., 2011).

Example 1 (Oscillator Death ${ }^{2}$ ) Consider the following system of two oscillators:

$\dot{\theta}_{1}=1+\sin \left(\theta_{1}+\theta_{2}\right)+\sin \left(2 \theta_{1}\right)$
$\dot{\theta}_{2}=2+2 \sin \left(\theta_{1}+\theta_{2}\right)+2 \sin \left(2 \theta_{2}\right)$

that is, with the above notations, $\omega_{1}=1, \omega_{2}=2, \Gamma=0$, $\gamma_{11}=\gamma_{12}=1, \gamma_{21}=\gamma_{22}=2$. For $\theta_{1}(0)=\theta_{2}(0)=\pi / 12$, it can be seen that $\dot{\theta}_{1}(t)=\dot{\theta}_{2}(t)=0$, for all $t \geq 0$. This corresponds to a non-oscillating phase-locked solution.

We note that a simple sufficient condition to avoid oscillator death (i.e. non-oscillating phase-locking) is given by

$\max _{i=1, \ldots, N}\left|\omega_{i}\right|>\max _{i=1, \ldots, N} \sum_{j=1, j \neq i}^{N}\left|k_{i j}+\gamma_{i j}\right|+\max _{i=1, \ldots, N} \sum_{j=1}^{N}\left|\gamma_{i j}\right|$

meaning that at least one natural frequency is sufficiently large with respect to the coupling and feedback gain. This condition ensures that the phase dynamics (4) does not have fixed points.

${ }^{2}$ We thank an anonymous reviewer of the conference version of this paper for bringing this example to our attention. 


\subsection{Existence of oscillating phase-locking}

We now present a general result on phase-locking under mean-field feedback. Its proof is given in Section 6.3.

Theorem 1 For almost all natural frequencies $\omega \in \mathbb{R}^{N}$, for almost all interconnection matrices $k \in \mathbb{R}^{N \times N}$, and for almost all feedback gains $\gamma \in \mathbb{R}^{N \times N}$, system (4) admits no oscillating phase-locked solution.

Theorem 1 states that, for a generic neuronal interconnection, the use of a proportional mean-field feedback prevents the oscillators to all evolve at the exact same frequency. Generically, under mean-field feedback, only two situations may therefore occur: either no phaselocking or no oscillations. This result therefore constitutes a promising feature of mean-field feedback DBS.

On the one hand, the strength of Theorem 1 stands in the generality of its assumptions: it holds for generic interconnections between neurons, including negative weights (inhibitory synapses), and does not require any knowledge neither on the contribution $\alpha_{j}$ of each neuron on the overall measurement nor on the intensity $\beta_{j}$ of the stimulation on each neuron. On the other hand, the disappearance of the phase-locked states does not prevent a pathological behavior. Indeed, while Theorem 1 states that the perfectly synchronized behavior is not compatible with mean-field feedback, it does not exclude the possibility of some kind of "practical" phase-locking, such as solutions whose mean behavior is near to that of a phase-locked one, but with small oscillations around it. For instance, they may correspond to phase differences which, while not remaining constant, stay bounded at all time. From a medical point of view, such a behavior for the neurons in the STN would anyway lead to tremor. We address this problem in Section 4. Moreover, as illustrated in Example 1 and Franci et al. (2011), a too large feedback gain may lead non-oscillating phase-locked solution, corresponding to neuronal inhibition.

Numerical simulations illustrate these features. The plots of (a) the phase differences with respect to their mean $\psi$ and (b) the order parameter $r_{\infty}$, given by $r_{\infty} e^{i \psi}:=\frac{1}{N} \sum_{j=1}^{N} e^{\mathrm{i} \theta_{j}}$, are provided for a large (Fig. 1 and 2) and a small (Fig. 3) feedback gain. Mean-field feedback is applied at $t=100$. While full desynchronization is achieved for the large feedback gain, practical phase-locking is observed in case of a too small feedback.

\subsection{Characterization of phase-locking}

The proof of Theorem 1, provided in Section 6.3, is based on two main steps, which are presented here as Lemmas 1 and 2 . Their interest goes beyond the technical aspects of the proof, as they underline some intrinsic properties
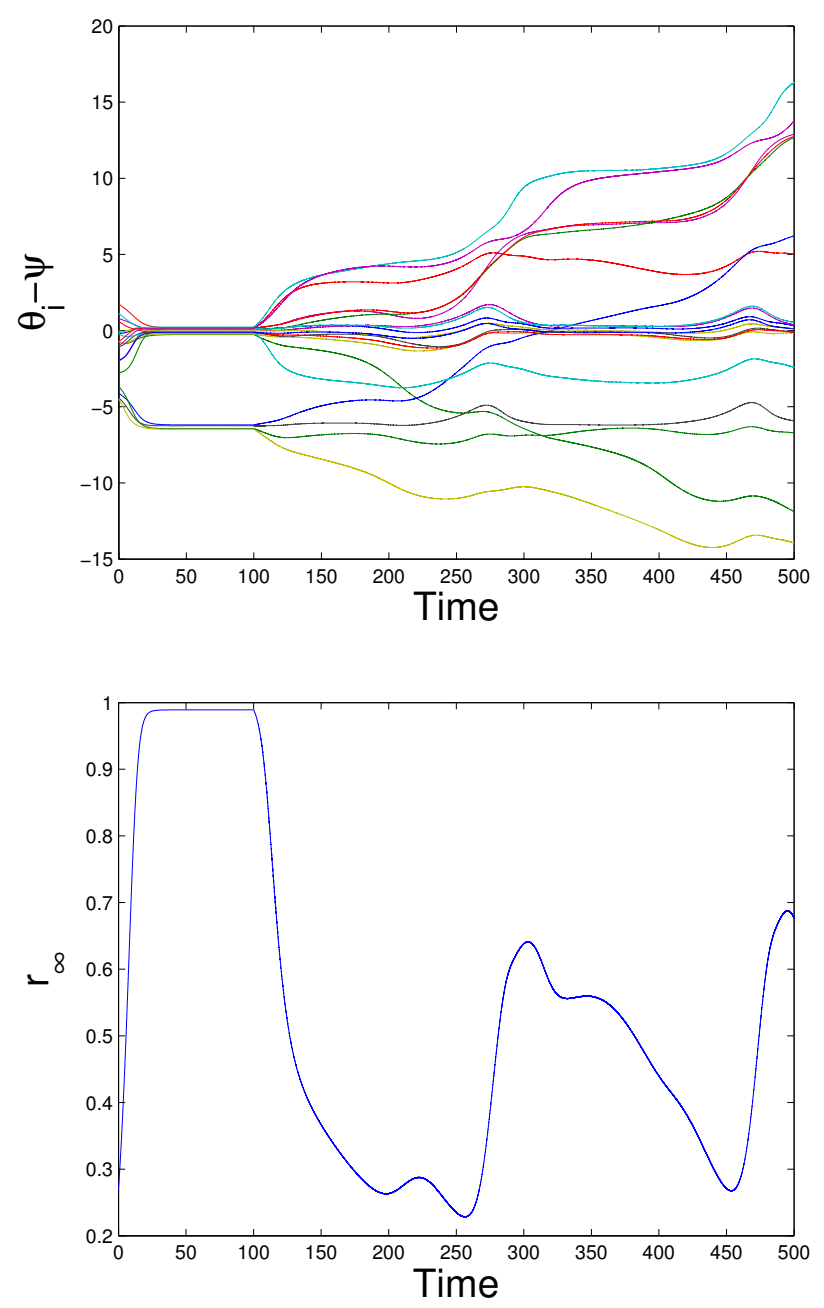

Fig. 1. Large feedback gain, full desynchronization.

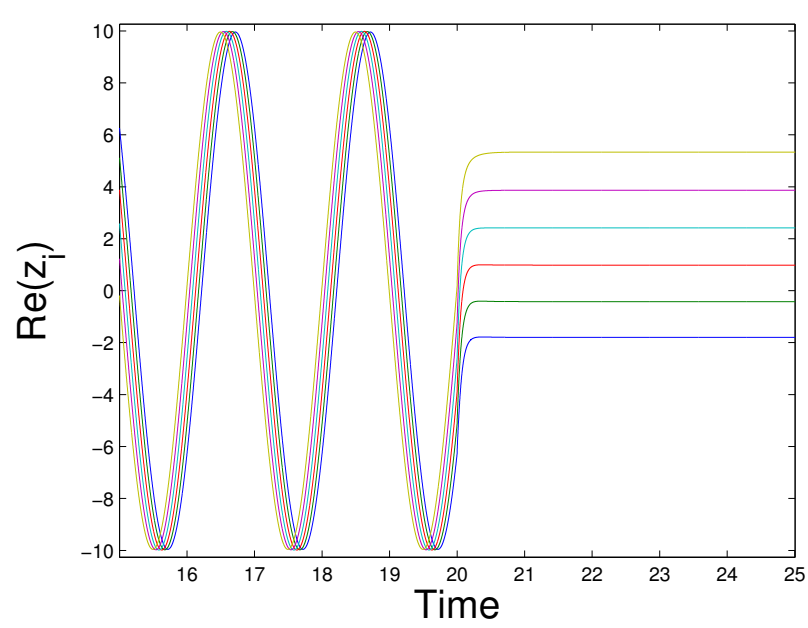

Fig. 2. Large feedback gain, neuronal inhibition. 

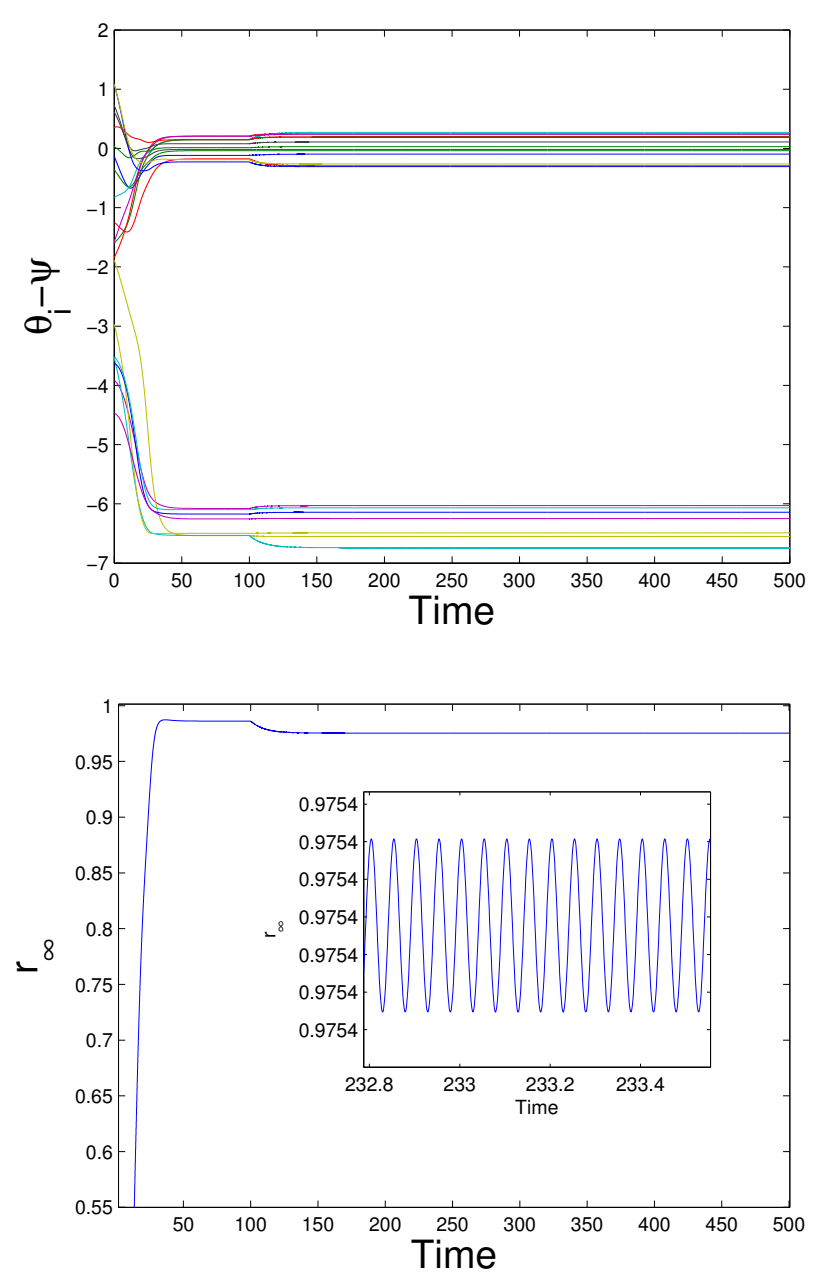

Fig. 3. Small feedback gain, practical phase-locking.

of the Kuramoto system under mean-field feedback, and permit to give a characterization of its phase-locked solutions in terms of an associated fixed point equation. Lemma 1 states that the problem of finding a phaselocked solution can be reduced to solving a set of nonlinear algebraic equations in terms of the phase differences $\Delta$ and the collective frequency of oscillation $\Omega$. Its proof is provided in Section 6.1.

Lemma 1 (Fixed-point equation) For all initial conditions $\theta^{*}(0) \in \mathbb{R}^{N}$, all natural frequencies $\omega \in \mathbb{R}^{N}$, all coupling matrices $k \in \mathbb{R}^{N \times N}$, and all feedback gains $\gamma \in \mathbb{R}^{N \times N}$, if system (4) admits an oscillating phaselocked solution starting in $\theta^{*}(0)$ with phase differences $\Delta$ and collective frequency of oscillation $\Omega$ satisfying (10)-
(11), then, for all $1 \leq i<j \leq N$, it holds that

$$
\begin{gathered}
\omega_{j}-\omega_{i}+\sum_{l=1}^{N}\left[\left(k_{j l}+\gamma_{j l}\right) \sin \left(\Delta_{j l}\right)-\left(k_{i l}+\gamma_{i l}\right) \sin \left(\Delta_{i l}\right)\right]=0, \\
\sum_{l=1}^{N}\left[\gamma_{j l} \sin \left(2 \int_{0}^{t} \Omega(s) d s+\Delta_{j l}+2 \theta_{j}^{*}(0)\right)\right. \\
\left.\quad-\gamma_{i l} \sin \left(2 \int_{0}^{t} \Omega(s) d s+\Delta_{i l}+2 \theta_{i}^{*}(0)\right)\right]=0 .
\end{gathered}
$$

While this fact is trivial for the Kuramoto system without inputs $($ i.e. $\gamma=0)$, its generalization to the presence of real mean-field feedback is not straightforward. The first set of equations (12a) can be seen as the classical fixed point equation for a Kuramoto system with natural frequencies $\omega$ and coupling matrix $\Gamma=k+\gamma$. It may or may not lead to the existence of a phase-locked solution (see (Jadbabaie et al., 2004) for necessary and sufficient conditions). The second set of equations (12b) is linked to the action of the mean-field feedback. It trivially holds if the feedback gain $\gamma$ is zero. Intuitively, we can expect that if the frequency of the collective oscillation $\Omega$ is not zero then (12b) admits no solution for any $\gamma \neq 0$. To illustrate this fact, consider the following example with only two neurons.

Example 2 (Two neurons) For $\mathrm{N}=2$, equation (12b) boils down to a single condition. Consider $\omega_{1} \neq \omega_{2}$, $\gamma_{i j}=1$ and $k_{i j}=2$ for all $i, j \in\{1,2\}$. In view of $(10)$, condition $(12 \mathrm{~b})$ reads $\sin \left(2 \int_{0}^{t} \Omega(s) d s+2 \theta_{2}^{*}(0)\right)-$ $\sin \left(2 \int_{0}^{t} \Omega(s) d s+2 \theta_{2}^{*}(0)+2 \Delta_{21}\right)=0$, or, by invoking the trigonometric identity $\sin a-\sin b=$ $2 \cos \left(\frac{a+b}{2}\right) \sin \left(\frac{a-b}{2}\right)$,

$$
2 \cos \left(2 \int_{0}^{t} \Omega(s) d s+2 \theta_{2}^{*}(0)+\Delta_{21}\right) \sin \left(\Delta_{12}\right)=0
$$

for all $t \geq 0$. In addition, (10) imposes that $\Delta_{21}=-\Delta_{12}$. Consequently (12a) reads $\omega_{1}-\omega_{2}=2 \sin \left(\Delta_{21}\right)$. Since $\omega_{1} \neq \omega_{2}$, it results that $\Delta_{21} \notin\{0, \pi\}$. We conclude that (12b) admits no solution for any $\Omega$ which is not zero almost everywhere.

The second main step in the proof of Theorem 1 confirms that indeed, if (12) admits a solution $(\Delta, \Omega)$, then $\Delta$ is fully determined by the "standard" part (12a) of this fixed point equation. In particular the following lemma, whose proof is given in Section 6.2, states that, around almost any solution of (12a), the phase differences that define a phase-locked configuration $\Delta$ can be locally expressed as a smooth function of the natural frequencies $\omega$ and of the interconnection matrix $\Gamma$. 
Lemma 2 (The Kuramoto fixed point equation is invertible) There exists a set $\mathcal{N} \subset \mathbb{R}^{N} \times \mathbb{R}^{N \times N}$, and a set $\mathcal{N}_{0} \subset \mathcal{N}$ satifying $\mu\left(\mathcal{N}_{0}\right)=0$, such that (12a) with natural frequencies $\omega^{*} \in \mathbb{R}^{N}$ and modified interconnection matrix $\Gamma^{*}:=k^{*}+\gamma^{*} \in \mathbb{R}^{N \times N}$ admits a solution $\Delta^{*} \in \mathbb{R}^{N \times N}$ if and only if $\left(\omega^{*}, \Gamma^{*}\right) \in \mathcal{N}$. Moreover, for all $\left(\omega^{*}, \Gamma^{*}\right) \in \mathcal{N} \backslash \mathcal{N}_{0}$, there exists a neighborhood $U$ of $\left(\omega^{*}, \Gamma^{*}\right)$, a neighborhood $W$ of $\Delta^{*}$, and a smooth function $f: U \rightarrow W$, such that, for all $(\omega, \Gamma) \in U$, $(\omega, \Gamma, \Delta:=f(\omega, \Gamma))$ is the unique solution of (12a) in $U \times W$.

We close this section by stressing a limitation of Theorem 1 , due to its generic nature. Some particular configurations may indeed allow for phase-locking even under a mean-field feedback stimulation. The following counterexample illustrates this fact, by showing that all-to-all homogeneous interconnections preserves phase-locking under mean-field feedback if all the neurons have the same natural frequencies. We stress that the heterogeneity of neurons and the complexity in their coupling make this example irrelevant for STN modeling.

Example 3 (Oscillating phase-locking despite mean-field feedback) Consider the system (4) with $\omega_{i}=\omega_{j}, k_{i j}=k$ and $\gamma_{i j}=\gamma \neq-k$ for all $i, j=1, \ldots, N$. This choice corresponds to the all-to-all coupling of identical Kuramoto oscillators, and is well studied in the literature, cf. e.g. (Kuramoto, 1984; Sepulchre et al., 2007; Sarlette, 2009; Brown et al., 2003). The system of equations (12a) then reads, for all $1 \leq 1<j \leq N$, $\sum_{l=1}^{N}(k+\gamma)\left(\sin \left(\Delta_{j l}\right)-\sin \left(\Delta_{i l}\right)\right)=0$. A solution to this equation is $\Delta_{i j}=0$ for all $1 \leq 1<j \leq N$. In view of (10), this is equivalent to the existence of $\theta_{0} \in \mathrm{T}^{1}$ such that $\theta_{i}^{*}(0)=\theta_{0}$, for all $i=1, \ldots, N$. The system of equations $(12 \mathrm{~b})$ then reads $\gamma \sum_{l=1}^{N}\left[\left(\sin \left(2 \int_{0}^{t} \Omega(s) d s+\right.\right.\right.$ $\left.\left.\left.\theta_{0}\right)-\sin \left(2 \int_{0}^{t} \Omega(s) d s+\theta_{0}\right)\right)\right]=0$, which is trivially satisfied for any collective frequency of oscillation $\Omega$. Lemma 2 thus ensures that oscillating phase-locking is preserved despite the mean-field stimulation.

\section{Robustness of phase-locked solutions}

As anticipated in Section 3, the disappearance of perfectly phase-locked states does not guarantee that the system is fully desynchronized. It may indeed happen that the system remains in a "practically" phase-locked state. This section aims at providing analytical justifications of this fact, by developing a robustness analysis of phase-locked solution in the Kuramoto system of coupled oscillators. The results presented in this section hold for all kinds of phase-locking (oscillating or not).

\subsection{Modeling exogenous inputs}

We start by slightly generalizing system (4) to take into account general time-varying inputs:

$$
\dot{\theta}_{i}(t)=\varpi_{i}(t)+\sum_{j=1}^{N} \tilde{k}_{i j} \sin \left(\theta_{j}(t)-\theta_{i}(t)\right),
$$

for all $t \geq 0$ and all $i=1, \ldots, N$, where $\varpi_{i}: \mathbb{R} \rightarrow \mathbb{R}$ denotes the input of the $i$-th oscillator, and $\tilde{k}=$ $\left[\tilde{k}_{i j}\right]_{i, j=1, \ldots, N} \in \mathbb{R}_{\geq 0}^{N \times N}$ is the coupling matrix. We stress that, in this section, only nonnegative interconnection gains are considered; negative gains are assumed minoritary and are trated as perturbations. Beyond the effect of the mean-field feedback, the system (13) encompasses the heterogeneity between the oscillators, the presence of exogenous disturbances and the uncertainties in the interconnection topology (time-varying coupling, negative interconnection gains, etc.). To see this clearly, let $\omega_{i}$ denote the natural frequency of the agent $i$, let $p_{i}$ represent its additive external perturbations, and let $\varepsilon_{i j}$ denote the uncertainty on each coupling gain $\tilde{k}_{i j}$. We assume that $p_{i}, \varepsilon_{i j}: \mathbb{R}_{\geq 0} \rightarrow \mathbb{R}$ are bounded piecewise continuous functions for each $i, j=1, \ldots, N$. Then the effects of all these disturbances, including mean-field feedback, can be analyzed in a unified manner by (13) by letting, for all $t \geq 0$ and all $i=1, \ldots, N$,

$$
\begin{aligned}
& \varpi_{i}(t)=\omega_{i}+p_{i}(t)+\sum_{j=1}^{N} \varepsilon_{i j}(t) \sin \left(\theta_{j}(t)-\theta_{i}(t)\right)+ \\
& \sum_{j=1}^{N}\left[\gamma_{i j} \sin \left(\theta_{j}(t)-\theta_{i}(t)\right)-\gamma_{i j} \sin \left(\theta_{j}(t)+\theta_{i}(t)\right)\right],
\end{aligned}
$$

which is well defined due to the forward completeness of (13). In Definition 1, the problem of finding a phaselocked solution has been translated into the search of a fixed point for the incremental dynamics (8). In the same spirit, the robustness analysis of phase-locked states is translated into some robustness properties of these fixed points. We define the common drift $\bar{\omega}$ of (13) as

$$
\bar{\omega}(t)=\frac{1}{N} \sum_{j=1}^{N} \varpi_{j}(t), \quad \forall t \geq 0
$$

and the grounded input as $\tilde{\omega}:=\left[\tilde{\omega}_{i}\right]_{i=1, \ldots, N}$, where

$$
\tilde{\omega}_{i}(t):=\varpi_{i}(t)-\bar{\omega}(t), \quad \forall i=1, \ldots, N, \forall t \geq 0 .
$$

Noticing that $\varpi_{i}-\varpi_{j}=\tilde{\omega}_{i}-\tilde{\omega}_{j}$, the evolution equation of the incremental dynamics ruled by (13) then reads

$$
\dot{\theta}_{i}(t)-\dot{\theta}_{j}(t)=\tilde{\omega}_{i}(t)-\tilde{\omega}_{j}(t)+
$$




$$
\sum_{l=1}^{N} \tilde{k}_{i l} \sin \left(\theta_{l}(t)-\theta_{i}(t)\right)-\sum_{l=1}^{N} \tilde{k}_{j l} \sin \left(\theta_{l}(t)-\theta_{j}(t)\right),
$$

for all $i, j=1, \ldots, N$ and all $t \geq 0$. As expected, the incremental dynamics is invariant to common drifts, which explain why the results of this section hold for all kinds of phase-locking (oscillating or not). In the sequel we use $\tilde{\theta}$ to denote the incremental variable

$$
\tilde{\theta}:=\left[\theta_{i}-\theta_{j}\right]_{i, j=1, \ldots, N, i \neq j} \in \mathrm{T}^{(N-1)^{2}} .
$$

\subsection{Robustness analysis}

When no inputs are applied, i.e. $\tilde{\omega} \equiv 0$, we expect the solutions of (17) to converge to some asymptotically stable fixed point or, equivalently, the solution of (13) to converge to some asymptotically stable phase-locked solution at least for some coupling matrices $k$. To make this precise, we start by defining the notion of 0-asymptically stable (0-AS) phase-locked solutions, which are described by asymptotically stable fixed points of the incremental dynamics (17) when no inputs are applied.

Definition 2 (0-AS phase-locked solutions) Given a coupling matrix $\tilde{k} \in \mathbb{R}_{\geq 0}^{N \times N}$, let $\mathcal{O}_{\tilde{k}}$ denote the set of all asymptotically stable fixed points of the unperturbed (i.e. $\tilde{\omega} \equiv 0$ ) incremental dynamics (17). A phase-locked solution $\theta^{*}$ of $(13)$ is said to be 0 asymptotically stable if and only if the incremental state $\tilde{\theta}^{*}:=\left[\theta_{i}^{*}-\theta_{j}^{*}\right]_{i, j=1, \ldots, N, i \neq j}$ belongs to $\mathcal{O}_{\tilde{k}}$.

A complete characterization of 0 -AS phase-locked solutions of (13) for general interconnection topologies can be found in (Sepulchre et al., 2008) and (Sarlette, 2009). In Section 4.5, we characterize the set $\mathcal{O}_{\tilde{k}}$ in terms of the isolated local minima of a suitable Lyapunov function. The reason for considering only asymptotically stable fixed points of the incremental dynamics lies in the fact that only those guarantee the robustness property of local Input-to-State Stability with respect to small inputs (Sontag and Wang, 1996), also referred to as Total Stability (Malkin, 1958; Loría and Panteley, 2005). On the contrary, (non 0-AS) stable fixed points may exhibit non-robust phase-locked states, as illustrated by the following example.

Example 4 (Non robust phase-locking) Consider the case where $N>2$ and let $\tilde{k}_{12}=\tilde{k}_{21}>0$, and $\tilde{k}_{i j}=0$ for all $(i, j) \in \mathbb{N}_{\leq N} \times \mathbb{N}_{\leq N} \backslash\{(1,2),(2,1)\}$. When $\tilde{\omega}=0$, the dynamics (17) reads

$$
\dot{\theta}_{i}-\dot{\theta}_{j}=0
$$

for all $(i, j) \in \mathbb{N}_{\leq N} \times \mathbb{N}_{\leq N} \backslash\{(1,2),(2,1)\}$, and

$$
\dot{\theta}_{1}-\dot{\theta}_{2}=-2 \tilde{k}_{12} \sin \left(\theta_{1}-\theta_{2}\right)
$$

In this case, all the solutions of the form $\theta_{1}(t)-\theta_{2}(t)=$ 0 , for all $t \geq 0$, and $\theta_{i}(t)-\theta_{j}(t)=\theta_{i}(0)-\theta_{j}(0)$, for all $t \geq 0$ and all $(i, j) \in \mathbb{N}_{\leq N} \times \mathbb{N}_{\leq N} \backslash\{(1,2),(2,1)\}$ are phase-locked. They can be shown to be stable, but not asymptotically. By adding any (arbitrarily small) constant inputs $\tilde{\omega}_{l} \neq 0$ to one of the agent $l \in \mathbb{N}_{\leq N} \backslash$ $\{1,2\}$, the system becomes completely desynchronized, since $\dot{\theta}_{l}-\dot{\theta}_{i} \equiv \tilde{\omega}_{l}$ for all $i=1, \ldots, N, i \neq l$. In particular, the set $\mathcal{O}_{\tilde{k}}$ is empty for this particular case.

Definition 3 (LISS w.r.t. small inputs) For a system $\dot{x}=f(x, u)$, a set $\mathcal{A} \subset \mathbb{R}^{n}$ is said to be locally input-to-state stable (LISS) with respect to small inputs iff there exist some constants $\delta_{x}, \delta_{u}>0$, a $\mathcal{K} \mathcal{L}$ function $\beta$ and a $\mathcal{K}_{\infty}$ function $\rho$, such that, for all $\left|x_{0}\right|_{\mathcal{A}} \leq \delta_{x}$ and all $u$ satisfying $\|u\| \leq \delta_{u}$, its solution satisfies

$$
\left|x\left(t, x_{0}, u\right)\right|_{\mathcal{A}} \leq \beta\left(\left|x_{0}\right|_{\mathcal{A}}, t\right)+\rho(\|u\|), \quad \forall t \geq 0 .
$$

If this holds with $\beta(r, s)=C r e^{-\frac{s}{\tau}}$ for all $r, s \geq 0$, where $C, \tau$ are positive constants, then $\mathcal{A}$ is locally exponentially Input-to-State Stable with respect to small inputs.

Remark 1 (Local Euclidean metric on the $n$ Torus) Definition 3 is given on $\mathbb{R}^{n}$, which is not well adapted to the context of this article. Its extension to the $n$-Torus is natural since $\mathrm{T}^{n}$ is locally isometric to $\mathbb{R}^{n}$ through the identity map $\mathcal{I}\left(\right.$ i.e. $\left.|\theta|_{\mathrm{T}^{n}}:=|\mathcal{I}(\theta)|=|\theta|\right)$. In particular this means that the $n$-Torus can be equipped with the local Euclidean metric and its induced norm. Hence, Definition 3 applies locally to the $n$-Torus.

The next theorem, whose proof is given in Section 6.6, states the LISS of $\mathcal{O}_{\tilde{k}}$ with respect to small inputs $\tilde{\omega}$.

Theorem 2 Let $\tilde{k} \in \mathbb{R}_{\geq 0}^{N \times N}$ be a given symmetric interconnection matrix. Suppose that the set $\mathcal{O}_{\tilde{k}}$ of Definition 3 is non-empty. Then the the set $\mathcal{O}_{\tilde{k}}$ is LISS with respect to small $\tilde{\omega}$ for (17). In other words, there exist $\delta_{\tilde{\theta}}, \delta_{\omega}>0, \beta \in \mathcal{K} \mathcal{L}$ and $\rho \in \mathcal{K}_{\infty}$, such that, for all $\tilde{\omega}$ and all $\tilde{\theta}_{0} \in T^{(N-1)^{2}}$ satisfying $\|\tilde{\omega}\| \leq \delta_{\omega}$ and $\left|\theta_{0}\right|_{\mathcal{O}_{\tilde{k}}} \leq \delta_{\tilde{\theta}}$,

$$
|\tilde{\theta}(t)|_{\mathcal{O}_{\tilde{k}}} \leq \beta\left(\left|\tilde{\theta}_{0}\right|_{\mathcal{O}_{\tilde{k}}}, t\right)+\rho(\|\tilde{\omega}\|), \quad \forall t \geq 0
$$

Theorem 2 guarantees that, if a given configuration is asymptotically stable for the unperturbed system, then solutions starting sufficiently close to that configuration remain near it at all time, in presence of sufficiently small perturbations $\tilde{\omega}$. Moreover, the steady-state distance of the incremental state $\tilde{\theta}$ from $\mathcal{O}_{\tilde{k}}$ is somewhat "proportional" to the amplitude of $\tilde{\omega}$ with nonlinear gain $\rho$. This means that the phase-locked states are robust to time-varying natural frequencies, provided that they are not too heterogeneous. We stress that, while local ISS with respect to small inputs is a natural consequence of 
asymptotic stability (Loría and Panteley, 2005), the size of the constants $\delta_{x}$ and $\delta_{u}$ in Definition 3, defining the robustness domain in terms of initial conditions and inputs amplitude, are potentially very small. As we show explicitly in the next section in the special case of all-toall coupling, the Lyapunov analysis used in the proof of Theorem 2 (cf. Section 6.6) provides a general methodology to build these estimates explicitly. In particular, while the region of attraction depends on the geometric properties of the fixed points of the unperturbed system, the size of admissible inputs can be made arbitrarily large by taking a sufficiently large coupling strength. This is detailed in the sequel (cf. (54), (55), (59) and (60) below).

\subsection{Robustness of the synchronized state in the case of all-to-all coupling}

In this section we focus the Lyapunov analysis used in the proof of Theorem 2 (cf. Section 6.6) to the case of all-to-all coupling. In this case, it is known (Sepulchre et al., 2008) that the only asymptotically stable phaselocked solution is the exact synchronization

$$
\tilde{\theta}(t)=0, \quad \forall t \geq 0
$$

corresponding to a zero phase difference between each pair of oscillators. The following proposition states the local exponential input-to-state stability of the synchronized state with respect to small inputs, and provides explicit estimates of the region of convergence, of the size of admissible inputs, of the ISS gain, and of the convergence rate. Its proof can be found in Section 6.8.

Proposition 1 (Exponential LISS of synchronization) Consider the system (13) with the all-to-all interconnection topology, i.e. $\tilde{k}_{i j}=K>0$ for all $i, j=$ $1, \ldots, N$. Then, for all $\epsilon \in\left[0, \frac{\pi}{2}\right]$, and all $\tilde{\omega}$ satisfying

$$
\|\tilde{\omega}\| \leq \delta_{\omega}^{\epsilon}:=\frac{K \sqrt{N}}{\pi^{2}}\left(\frac{\pi}{2}-\epsilon\right)
$$

the following facts hold:

(1) the set $\mathcal{D}_{\epsilon}:=\left\{\tilde{\theta} \in T^{(N-1)^{2}}:|\tilde{\theta}|_{\infty} \leq \frac{\pi}{2}-\epsilon\right\}$ is forward invariant for the system (17);

(2) for all $\tilde{\theta}_{0} \in \mathcal{D}_{0}$, the set $\mathcal{D}_{\epsilon}$ is attractive, and the solution of (17) satisfies

$$
|\tilde{\theta}(t)| \leq \frac{\pi}{2}\left|\tilde{\theta}_{0}\right| e^{-\frac{K}{\pi^{2}} t}+\frac{\pi^{2}}{K}\|\tilde{\omega}\|, \quad \forall t \geq 0 .
$$

Proposition 1 establishes the exponential ISS of the synchronized state in the all-to-all Kuramoto model with respect to time-varying inputs whose amplitudes are smaller than $\frac{K \sqrt{N}}{2 \pi}$. It holds for any initial condition lying in $\mathcal{D}_{0}$, that is when all the initial phase differences lie in $\left[-\frac{\pi}{2}, \frac{\pi}{2}\right]$. Moreover, if the inputs amplitude is bounded by $\delta_{\omega}^{\epsilon}$, for some $0 \leq \epsilon \leq \frac{\pi}{2}$, then the set $\mathcal{D}_{\epsilon}$ is forward invariant and all the solutions starting in $\mathcal{D}_{0}$ actually converge to $\mathcal{D}_{\epsilon}$.

Recently, necessary and sufficient conditions for the exponential synchronization of the Kuramoto system with all-to-all coupling and constant different natural frequencies were given in (Chopra and Spong, 2009). We stress that the estimated region of attraction provided by Proposition 1 is strictly larger than the one obtained in (Chopra and Spong, 2009, Theorem 4.1), which does not allow $\epsilon$ to be picked as zero. For initial conditions lying in $\mathcal{D}_{\epsilon}$, with a strictly positive $\epsilon$, it is interesting to compare the convergence rate obtained in Proposition $1, \frac{K}{\pi^{2}}$, with the one obtained in (Chopra and Spong, 2009, Theorem 3.1), NK $\sin (\epsilon)$. While the convergence rate of Proposition 1 is slower than the one obtained in (Chopra and Spong, 2009, Theorem 3.1) for large $\epsilon$, it provides a better estimate for small values of $\epsilon$. Furthermore, for any fixed amplitude $\|\tilde{\omega}\|$, the bound (21) allows to find the sufficient coupling strength $K_{\epsilon}$ which ensures the attractivity of $\mathcal{D}_{\epsilon}$ :

$$
K_{\epsilon}=\frac{\pi^{2}}{\left(\frac{\pi}{2}-\epsilon\right) \sqrt{N}}\|\tilde{\omega}\| .
$$

Noticing that $\sqrt{N} \max _{i, j=1, \ldots, N}\left\|\varpi_{i}-\varpi_{j}\right\| \geq\|\tilde{\omega}\|$, we get that

$$
K_{\epsilon} \leq \frac{\pi^{2}}{\left(\frac{\pi}{2}-\epsilon\right)} \max _{i, j=1, \ldots, N}\left\|\varpi_{i}-\varpi_{j}\right\| .
$$

Since $\left(\frac{\pi}{2}-\epsilon\right) \geq \frac{2}{\pi} \cos (\epsilon)$, for all $0 \leq \epsilon \leq \frac{\pi}{2}$, it results that

$$
K_{\epsilon} \leq \frac{\pi^{3}}{2 \cos (\epsilon)} \max _{i, j=1, \ldots, N}\left\|\varpi_{i}-\varpi_{j}\right\|<\pi^{3} K_{i n v}
$$

where $K_{i n v}$ is the sufficient coupling strength provided in (Chopra and Spong, 2009, Proof of Theorem 4.1). This observation shows that, while the estimate $K_{\epsilon}$ may be more restrictive than the one proposed in (Chopra and Spong, 2009), both are of of the same order, in the sense that $\frac{K_{\epsilon}}{K_{i n v}}<\pi^{3}$. For the same region of attraction, a tighter bound $K_{\text {suff }}$ for the sufficient coupling strength has recently been given in (Dörfler and Bullo, 2011), where this bound is inversely proportional to the number of oscillators, that is $\frac{K_{\epsilon}}{K_{s u f f}} \sim N$. Nonetheless, similarly to (Chopra and Spong, 2009), their rate of convergence is proportional to $\sin (\epsilon)$, leading to a worse bound than ours for large regions of attraction. In conclusion, Proposition 1 partially extends the main results of (Chopra and Spong, 2009; Dörfler and Bullo, 2011) to time-varying inputs. On the one hand, it allows to consider sets of initial conditions larger than those of 
(Chopra and Spong, 2009), and bounds the convergence rate by a strictly positive value, independently of the region of attraction. On the other hand the required coupling strength is comparable to the one given in (Chopra and Spong, 2009), but more conservative than the lower bound in (Dörfler and Bullo, 2011). Finally for small regions of attraction, the bound on the convergence rate obtained in Proposition 1 is not as good as the one of (Chopra and Spong, 2009; Dörfler and Bullo, 2011).

\subsection{Robustness of neural synchrony to mean-field feed- back Deep Brain Stimulation}

As a corollary of Theorem 2 we derive necessary conditions on the intensity of a desynchronizing mean-field feedback. To that end consider the Kuramoto system under mean-field feedback (4), and let $\bar{\gamma}$ and $\omega^{\perp}$ represent the intensity of the mean-field feedback DBS and the heterogeneity of the ensemble of neurons:

$$
\begin{gathered}
\bar{\gamma}:=\max _{i, j=1, \ldots, N}\left|\gamma_{i j}\right|, \\
\omega^{\perp}:=\left[\omega_{i}-\frac{1}{N} \sum_{j=1}^{N} \omega_{j}\right]_{i=1, \ldots, N},
\end{gathered}
$$

We also define the grounded mean-field input $\tilde{I}_{M F}$ of the incremental dynamics associated to (4) as

$$
\tilde{I}_{M F}(t):=I_{M F}(t)-\bar{I}_{M F}(t) \overrightarrow{1}_{N}, \quad \forall t \geq 0,
$$

where, for all $t \geq 0, I_{M F}(t):=\left[I_{M F_{i}}(t)\right]_{i=1, \ldots, N}$,

$$
I_{M F_{i}}(t):=\sum_{j=1}^{N} \gamma_{i j}\left(\sin \left(\theta_{i}(t)-\theta_{j}(t)\right)-\sin \left(\theta_{i}(t)+\theta_{j}(t)\right)\right.
$$

is the input of the mean-field feedback (cf. (14)) and

$\bar{I}_{M F}(t):=\frac{1}{N} \sum_{i, j=1}^{N} \gamma_{i j}\left(\sin \left(\theta_{i}(t)-\theta_{j}(t)\right)-\sin \left(\theta_{i}(t)+\theta_{j}(t)\right)\right.$

for all $t \geq 0$, represents the common drift among the ensemble of neurons due to the mean-field feedback. The following result, whose proof is given in Section 6.7, stresses the robustness of phase-locking with respect to mean-field feedback. It provides a negative answer to the question whether mean-field feedback DBS with arbitrarily small amplitude can effectively desynchronize.

Corollary 1 (Robustness of phase-locking to mean-field feedback) Let $k \in \mathbb{R}_{\geq 0}^{N \times N}$ be a given symmetric interconnection matrix and $\omega \in \mathbb{R}^{N}$ be any (constant) vector of natural frequencies. Let $\gamma \in \mathbb{R}^{N \times N}$ be any feedback gain. Let $\bar{\gamma}, \omega^{\perp}$, and $\tilde{I}_{M F}$ be defined as in (22)-(24). Let the set $\mathcal{O}_{k}$ be defined as in Definition 2 and suppose that it is non-empty. Then there exist a class $\mathcal{K} \mathcal{L}$ function $\beta$, a class $\mathcal{K}_{\infty}$ function $\sigma$, a positive constant $\delta_{\omega}$, and a neighborhood $\mathcal{P}$ of $\mathcal{O}_{k}$, such that, for all natural frequencies and all mean-field feedback satisfying

$$
\left|\omega^{\perp}\right|+2 \bar{\gamma} N \sqrt{N} \leq \delta_{\omega}
$$

the solution of (4) satisfies, for all $\tilde{\theta}_{0} \in \mathcal{P}$,

$$
|\tilde{\theta}(t)|_{\mathcal{O}_{k}} \leq \beta\left(\left|\tilde{\theta}_{0}\right|_{\mathcal{O}_{k}}, t\right)+\sigma\left(\left|\omega^{\perp}\right|+\left\|\tilde{I}_{M F}\right\|\right), \forall t \geq 0,
$$

where $\tilde{\theta}$ is defined in (18).

Corollary 1 states that the phase-locked states associated to any symmetric interconnection topology are robust to sufficiently small mean-field feedbacks. The intensity of the tolerable feedback gain $\bar{\gamma}$ depends on the distribution of natural frequencies, reflecting the fact that a heterogeneous ensemble can be more easily brought to an incoherent state.

\subsection{A Lyapunov function for the incremental dynamics}

In this section, we introduce the Lyapunov function for the incremental dynamics (17), that will be referred to as the incremental Lyapunov function. It will be used in the proof of Theorem 2 . We start by showing that the incremental dynamics (17) possesses an invariant manifold, that we characterize through some linear relations. This observation allows us to restrict the analysis of the critical points of the Lyapunov function to this manifold. Beyond its technical interest, this analysis shows that phase-locked solutions correspond to these critical points. In particular, it provides an analytic way of computing the set $\mathcal{O}_{\tilde{k}}$ of Definition 2, completely characterizing the set of robust asymptotically stable phase-locked solutions.

\section{The incremental Lyapunov function}

We start by introducing the normalized interconnection matrix associated to $\tilde{k}$

$$
E=\left[E_{i j}\right]_{i, j=1, \ldots, N}:=\frac{1}{K}\left[\tilde{k}_{i j}\right]_{i, j=1, \ldots, N}
$$

where the constant $K>0$ is defined as

$$
K:=\left\{\begin{array}{ccc}
1 & \text { if } & \tilde{k}=0 \\
\max _{i, j=1, \ldots, N} \tilde{k}_{i j} & \text { if } & \tilde{k} \neq 0 .
\end{array}\right.
$$


Inspired by (Sarlette, 2009), let $V_{I}: \mathrm{T}^{(N-1)^{2}} \rightarrow \mathbb{R}_{\geq 0}$ be the incremental Lyapunov function defined by

$$
V_{I}(\tilde{\theta}):=2 \sum_{i=1}^{N} \sum_{j=1}^{N} E_{i j} \sin ^{2}\left(\frac{\theta_{i}-\theta_{j}}{2}\right),
$$

where the incremental variable $\tilde{\theta}$ is defined in (18). We stress that $V_{I}$ is independent of the coupling strength $K$.

\section{The invariant manifold}

The presence of an invariant manifold results from the fact that the components of the incremental variable $\tilde{\theta}$ are not linearly independent. Indeed, we can express $(N-1)(N-2)$ of them in terms of the other $N-1$ independent components. More precisely, by choosing $\varphi_{i}:=\theta_{i}-\theta_{N}, i=1, \ldots, N-1$, as the independent variables, it is possible to write, for all $i=1, \ldots, N-1$,

$$
\begin{aligned}
\theta_{i}-\theta_{N} & =\varphi_{i}, \\
\theta_{i}-\theta_{j} & =\varphi_{i}-\varphi_{j}, \quad \forall j=1, \ldots, N-1 .
\end{aligned}
$$

These relations can be expressed in a compact form as

$$
\tilde{\theta}=\tilde{B}(\varphi):=B \varphi \quad \bmod 2 \pi, \quad \varphi \in \mathcal{M}
$$

where $\varphi:=\left[\varphi_{i}\right]_{i=1, \ldots, N-1}, \quad B \in \mathbb{R}^{(N-1)^{2} \times(N-1)}$, $\operatorname{rank} B=N-1, \tilde{B}$ is continuously differentiable, and $\mathcal{M} \subset \mathrm{T}^{(N-1)^{2}}$ is the submanifold defined by the embedding (29). The continuous differentiability of $\tilde{B}: \mathcal{M} \rightarrow \mathrm{T}^{(N-1)^{2}}$ comes from the fact that $\varphi_{i} \in \mathrm{T}^{1}$, for all $i=1, \ldots, N-1$, and from the additive group structure of $\mathrm{T}^{1}$. Formally, this means that the system is evolving on the manifold $\mathcal{M} \subset \mathrm{T}^{(N-1)^{2}}$ of dimension $N-1$. In particular $\mathcal{M}$ is diffeomorphic to $\mathrm{T}^{N-1}$.

\section{Restriction to the invariant manifold}

In order to conduct a Lyapunov analysis based on $V_{I}$ it is important to identify its critical points. Since the system is evolving on the invariant manifold $\mathcal{M}$, only the critical points of the Lyapunov function $V_{I}$ restricted to this manifold are of interest. Hence we focus on the critical points of the restriction of $V_{I}$ to $\mathcal{M}$, which is defined by the function $\left.V_{I}\right|_{\mathcal{M}}: \mathrm{T}^{N-1} \rightarrow \mathbb{R}_{\geq 0}$ as

$$
\left.V_{I}\right|_{\mathcal{M}}(\varphi):=V_{I}(B \varphi), \quad \forall \varphi \in \mathcal{M}
$$

The analysis of the critical points of $\left.V_{I}\right|_{\mathcal{M}}$ is not trivial. To simplify this problem, we exploit the fact that the variable $\varphi$ can be expressed in terms of $\theta$ by means of a linear transformation $A \in \mathbb{R}^{(N-1) \times N}$, with $\operatorname{rank} A=$ $N-1$, in such a way that

$$
\varphi=\tilde{A}(\theta):=A \theta \bmod 2 \pi
$$

Based on this, we define the function $V: \mathrm{T}^{N} \rightarrow \mathbb{R}$ as

$$
V(\theta):=\left.V_{I}\right|_{\mathcal{M}}(A \theta), \quad \forall \theta \in T^{N} .
$$

In contrast with $\left.V_{I}\right|_{\mathcal{M}}$, the function $V$ owns the advantage that its critical points are already widely studied in the synchronization literature, see for instance (Sepulchre et al., 2007) and (Sarlette, 2009, Chapter 3). The following lemma allows to reduce the analysis of the critical points of $V_{I}$ on $\mathcal{M}$ to that of the critical points of $V$ on $T^{N}$. Its proof is given in Section 6.4.

Lemma 3 (Computation of the critical points on the invariant manifold) Let $\mathcal{M},\left.V_{I}\right|_{\mathcal{M}}, A$ and $V$ be defined by (29)-(32). Then $\theta^{*} \in T^{N}$ is a critical point of $V$ (i.e. $\nabla_{\theta} V\left(\theta^{*}\right)=0$ ) if and only if $\varphi^{*}=A \theta^{*} \in \mathcal{M}$ is a critical point of $\left.V_{I}\right|_{\mathcal{M}}$ (i.e. $\left.\left.\nabla_{\varphi} V_{I}\right|_{\mathcal{M}}\left(\varphi^{*}\right)=0\right)$. Moreover if $\theta^{*}$ is a local maximum (resp. minimum) of $V$ then $\varphi^{*}$ is a local maximum (resp. minimum) of $\left.V_{I}\right|_{\mathcal{M}}$. Finally the origin of $\mathcal{M}$ is a local minimum of $\left.V_{I}\right|_{\mathcal{M}}$.

\section{Lyapunov characterization of robust phase-locking}

The above development allows to characterize phaselocked states through the incremental Lyapunov function $V_{I}$. The following lemma, proved in Section 6.5, states that the fixed points of the unperturbed incremental dynamics are the critical points of $\left.V_{I}\right|_{\mathcal{M}}$, modulo the linear relations $(28)$, i.e. the critical points of $\left.V_{I}\right|_{\mathcal{M}}$ completely characterize phase-locked solutions.

Lemma 4 (Incremental Lyapunov characterization of phase-locking) Let $k \in \mathbb{R}_{\geq 0}^{N \times N}$ be a given symmetric interconnection matrix. Let $B$ and $\left.V_{I}\right|_{\mathcal{M}}$ be defined as in (29) and (30). Then $\varphi^{*} \in \mathcal{M}$ is a critical point of $\left.V_{I}\right|_{\mathcal{M}}$ (i.e. $\left.\nabla_{\varphi} V_{I}\right|_{\mathcal{M}}\left(\varphi^{*}\right)=0$ ) if and only if $B \varphi^{*}$ is a fixed point of the unperturbed (i.e. $\tilde{\omega} \equiv 0$ ) incremental dynamics (17).

Remark 2 (Incremental Lyapunov characterization of robust 0-AS phase-locked solutions) When no inputs apply (i.e., $\tilde{\omega} \equiv 0$ ), the Lyapunov function $V_{I}$ is strictly decreasing along the trajectories of (17) if and only if the state does not belong to the set of critical points of $\left.V_{I}\right|_{\mathcal{M}}$ (cf. Claim 2 below). It then follows directly from Lemma 4 that isolated local minima of $\left.V_{I}\right|_{\mathcal{M}}$ correspond to asymptotically stable fixed points of (17). By Definition 2 and Theorem 2, we conclude that the robust asymptotically stable phase-locked states are completely characterized by the set of isolated local minima of $\left.V_{I}\right|_{\mathcal{M}}$. The computation of this set is simplified through Lemma 3.

\section{Consequence for the system without inputs}

At the light of Lemma 4, we can state the following corollary, which recovers, and partially extends, the result of 
(Sarlette, 2009, Proposition 3.3.2) in terms of the incremental dynamics of the system. It states that, for a symmetric interconnection topology, any disturbance with zero grounded input (16) preserves the almost global asymptotic stability of phase-locking for (13).

Corollary 2 (Almost global asymptotic phaselocking) Let $\varpi: \mathbb{R}_{\geq 0} \rightarrow \mathbb{R}^{N}$ be any signal satisfying $\tilde{\omega}(t)=0$, for all $t \geq 0$, where $\tilde{\omega}$ is defined in (16). If the interconnection matrix $\tilde{k} \in \mathbb{R}_{\geq 0}^{N \times N}$ is symmetric, then almost all trajectories of (13) converge to a stable phase-locked solution.

We stress that Corollary 2 is an almost global result. It follows from the fact that almost all trajectories converge to the set of local minima of $\left.V_{I}\right|_{\mathcal{M}}$. From Lemma 4, this set corresponds to asymptotically stable fixed points of the incremental dynamics, that is to asymptotically stable phase-locked solutions. The precise proof is omitted here.

\section{Conclusion and perspectives}

Motivated by neurological treatment applications we have shown that, generically, no network of Kuramoto oscillators can exhibit oscillating phase-locked solutions when a proportional mean-field feedback is applied. While this gives good hopes for effective output feedback desynchronization, the robustness analysis carried out in this paper also shows that too small feedback gains cannot be expected to fully decorrelate the oscillators. On-going work now aims at providing tuning methods for specific interconnections topologies in order to achieve either complete desynchronization or oscillator death. Future works will aim at extending these results to more detailed models. From a medical point of view, only future interdisciplinary studies will show how relevant the results of the article are regarding the realistic scenario of DBS. In particular, the practical possibility to measure and stimulate at the same time has to be carefully studied.

\section{Proofs}

\section{Proofs of the Results of Section 3}

\subsection{Proof of Lemma 1}

From (10)-(11), phase-locked solutions of (4) satisfy, for all $t \geq 0$ and all $i, j=1, \ldots, N$,

$$
\begin{aligned}
\theta_{j}^{*}(t)+\theta_{i}^{*}(t) & =\theta_{j}^{*}(t)-\theta_{i}^{*}(t)+2 \theta_{i}^{*}(t) \\
& =2 \Lambda_{\Omega}(t)+\Delta_{i j}+2 \theta_{i}^{*}(0),
\end{aligned}
$$

where $\Lambda_{\Omega}(t):=\int_{0}^{t} \Omega(s) d s$, for all $t \geq 0$. In view of (8), (10)-(11), and noting that the fixed point equation equation is trivial if $i=j$ and that, due to the antisymmetric dependence of (12) on $i$ and $j$, finding a solution for some $i=i^{*}$ and $j=j^{*}$ gives a solution also for $i=j^{*}$ and $j=i^{*}$, finding a phase-locked solution is equivalent to solving the set of equations

$$
\begin{aligned}
\omega_{j}-\omega_{i}+ & \sum_{l=1}^{N}\left[\left(k_{j l}+\gamma_{j l}\right) \sin \left(\Delta_{j l}\right)-\left(k_{i l}+\gamma_{i l}\right) \sin \left(\Delta_{i l}\right)\right]- \\
& \sum_{l=1}^{N}\left[\gamma_{j l} \sin \left(2 \Lambda_{\Omega}(t)+\Delta_{j l}+2 \theta_{j}^{*}(0)\right)\right. \\
& \left.-\gamma_{i l} \sin \left(2 \Lambda_{\Omega}(t)+\Delta_{i l}+2 \theta_{i}^{*}(0)\right)\right]=0
\end{aligned}
$$

for all $t \geq 0$ and all $1 \leq i<j \leq N$, in terms of $\Delta$ and $\Omega$. Note that the first line of the last equation is constant, that is there exist constants $\left\{c_{i j}\right\}_{i, j=1, \ldots, N, i<j}$ such that

$\omega_{j}-\omega_{i}+\sum_{l=1}^{N}\left[\left(k_{j l}+\gamma_{j l}\right) \sin \left(\Delta_{j l}\right)-\left(k_{i l}+\gamma_{i l}\right) \sin \left(\Delta_{i l}\right)\right]=c_{i j}$.

Equation (34) then reads

$$
\begin{gathered}
\sum_{l=1}^{N}\left[\gamma_{j l} \sin \left(2 \Lambda_{\Omega}(t)+\Delta_{j l}+2 \theta_{j}^{*}(0)\right)\right. \\
\left.-\gamma_{i l} \sin \left(2 \Lambda_{\Omega}(t)+\Delta_{i l}+2 \theta_{i}^{*}(0)\right)\right]=c_{i j},
\end{gathered}
$$

for all $t \geq 0$ and all $1 \leq i<j \leq N$. Hence, if a phaselocked solution exists, then there must exist a solution to the set of equations (35) and (36) in terms of $\Delta, \Omega$ and $c:=\left\{c_{i j}\right\}_{i, j=1, \ldots, N, i<j}$. By time-differentiating (36), one gets, for all $t \geq 0$ and all $1 \leq i<j \leq N$,

$$
\begin{gathered}
2 \Omega(t) \sum_{l=1}^{N}\left[\gamma_{j l} \cos \left(2 \Lambda_{\Omega}(t)+\Delta_{j l}+2 \theta_{j}^{*}(0)\right)\right. \\
\left.-\gamma_{i l} \cos \left(2 \Lambda_{\Omega}(t)+\Delta_{i l}+2 \theta_{i}^{*}(0)\right)\right]=0 .
\end{gathered}
$$

Since $\Omega$ is a non identically zero continuous function, there exists an open interval $(\underline{t}, \bar{t}), 0 \leq \underline{t}<\bar{t}$, such that $\Omega(t) \neq 0$ for all $t \in(\underline{t}, \bar{t})$. Hence $(37)$ implies that

$$
\begin{aligned}
& \sum_{l=1}^{N}\left[\gamma_{j l} \cos \left(2 \Lambda_{\Omega}(t)+\Delta_{j l}+2 \theta_{j}^{*}(0)\right)\right. \\
& \left.-\gamma_{i l} \cos \left(2 \Lambda_{\Omega}(t)+\Delta_{i l}+2 \theta_{i}^{*}(0)\right)\right]=0
\end{aligned}
$$

for all $t \in(\underline{t}, \bar{t})$. By differentiating (38) with respect to time and considering once again that $\Omega(t) \neq 0$ for all $t \in(\underline{t}, \bar{t})$, one gets that (36) holds true for all $t \in(\underline{t}, \bar{t})$, 
and for all $1 \leq i<j \leq N . c_{i j}$ being a constant this implies that, for all $t \geq 0, c_{i j}=0$, for all $1 \leq i<j \leq N$.

\subsection{Proof of Lemma 2}

For notational purposes, define $y_{i}:=\Delta_{i N}, i=$ $1, \ldots, N-1$, and $y:=\left[y_{i}\right]_{i=1, \ldots, N-1}$. Since it holds that $\Delta_{m n}=y_{n}-y_{m}$, for all $m, n=1, \ldots, N$, we can express all the phase differences in (12a) in terms of the components of $y$. Analogously, since $\dot{\theta}_{n}-\dot{\theta}_{m}=\dot{\theta}_{n}-\dot{\theta}_{N}-\left(\dot{\theta}_{m}-\dot{\theta}_{N}\right)$, it suffices to find a solution to the set of equation in (12a), relative to the pairs of index $(i, N), i=1, \ldots, N-1$. For the vectors $y$, we use the suffix ${ }^{*}$ when it refers to $\Delta^{*}$. Define, for $i=1, \ldots, N-1$,

$$
\begin{aligned}
& F_{i}(\omega, \Gamma, y):=\omega_{N}-\omega_{i}- \\
& \sum_{k=1}^{N-1}\left[\Gamma_{i k} \sin \left(y_{k}-y_{i}\right)-\Gamma_{N k} \sin y_{k}\right]-\Gamma_{i N} \sin y_{i} .
\end{aligned}
$$

With this notation, the equations in (12a) relative to the pairs of index $(i, N), i=1, \ldots, N-1$, can be written as

$$
F(\omega, \Gamma, y)=0 .
$$

In order to solve (40)in the form $y=f(\Gamma, \omega)$ through the implicit function theorem, we have to show that the matrix

$$
J(\Gamma, y):=\left[\frac{\partial F_{i}}{\partial y_{j}}(\Gamma, y)\right]_{i, j=1, \ldots, N-1},
$$

has full rank on the solutions of (40). Define, for $y \in$ $\mathbb{R}^{N-1}$,

$$
S(y):=\left\{\Gamma \in \mathbb{R}^{N \times N}: \operatorname{det} J(\Gamma, y)=0\right\} .
$$

Since det $J(\Gamma, y)$ is a non-identically zero analytical function (for example it is non-zero for $y=0$ and $\Gamma_{i j}=1$, for all $i, j=1, \ldots, N)$, it is zero only on sets of zero measure (Krantz and Parks, 2002, Chapter 4). In particular $\mu(S(y))=0$, for all $y \in \mathbb{R}^{N-1}$. Let the sets $\mathcal{N}$ and $\mathcal{N}_{0}$ be defined as

$$
\begin{aligned}
\mathcal{N}:=\left\{(\omega, \Gamma) \in \mathbb{R}^{N} \times \mathbb{R}^{N \times N}:\right. & \\
& \left.\exists y \in \mathbb{R}^{N-1}: F(\omega, \Gamma, y)=0\right\}
\end{aligned}
$$

and

$$
\begin{aligned}
\mathcal{N}_{0}: & =\left\{(\omega, \Gamma) \in \mathbb{R}^{N} \times \mathbb{R}^{N \times N}:\right. \\
& \left.\exists y \in \mathbb{R}^{N-1}: F(\omega, \Gamma, y)=0, \Gamma \in S(y)\right\},
\end{aligned}
$$

that is $\mathcal{N}$ contains all the natural frequencies and modified interconnection matrices that admit a solution to (12a), and $\mathcal{N}_{0}$ all the natural frequencies and modified interconnection matrices that admit a solution to (12a) such that (41) is singular. The next claim, proved in Section 7.1 , shows that $\mathcal{N}_{0}$ is of zero Lebesgue measure.

Claim 1 Let $\mathcal{N}_{0}$ be defined as in (44). Then $\mu\left(\mathcal{N}_{0}\right)=0$.

The lemma then follows directly from the implicit function theorem. Indeed, given any point $\left(\omega^{*}, \Gamma^{*}\right) \in \mathcal{N} \backslash \mathcal{N}_{0}$, since $\Gamma^{*} \notin S\left(y^{*}\right)$, the matrix $J\left(\Gamma^{*}, y^{*}\right)$, defined in (41), is invertible, and the existence of the neighborhoods $U$ and $W$, and of the function $f$ with the properties of the statement of the lemma follows directly from the implicit function theorem (Lee, 2006, Theorem 7.9).

\subsection{Proof of Theorem 1}

The proof consists in explicitly constructing a zero Lebesgue measure set of natural frequencies and coupling and feedback gains, out of which the system of equations (12) admits no solutions. The theorem then follows from Lemma 1.

Since the interconnection matrix $k$, the modified interconnection matrix $\Gamma$, and the feedback gain $\gamma$ are linked by the linear relation $k=\Gamma-\gamma$, we can independently fix $\Gamma$ and $\gamma$, and set $k$ accordingly. For all $\omega \in \mathbb{R}$, let

$$
\mathcal{M}_{0}(\omega):=\left\{\Gamma \in \mathbb{R}^{N \times N}:(\omega, \Gamma) \in \mathcal{N}_{0}\right\}
$$

where $\mathcal{N}_{0} \subset \mathbb{R}^{N} \times \mathbb{R}^{N \times N}$ is defined in the statement of Lemma 2 and has zero Lebesgue measure. Let

$$
\mathcal{S}_{0}:=\left\{\omega \in \mathbb{R}^{N}: \mu\left(\mathcal{M}_{0}(\omega)\right)>0\right\}
$$

If $\mu\left(\mathcal{S}_{0}\right)>0$, then $\mu\left(\mathcal{N}_{0}\right)=\int_{\mathcal{S}_{0}} \mu\left(\mathcal{M}_{0}(\omega)\right) d \omega>0$, which contradicts Lemma 2. Hence $\mu\left(\mathcal{S}_{0}\right)=0$.

Consider any $\omega \in \mathbb{R}^{N} \backslash \mathcal{S}_{0}$ and any $\Gamma \in \mathbb{R}^{N \times N} \backslash \mathcal{M}_{0}(\omega)$. In view of what precedes, this constitutes a generic choice or $\omega$ and $\Gamma$ and it holds that $(\omega, \Gamma) \notin \mathcal{N}_{0}$. Suppose that there exists an oscillating phase-locked solution starting in $\theta^{*}(0)$, with phase differences $\Delta$ and collective frequency of oscillation $\Omega$. From Lemma 1, a necessary condition for the existence of an oscillating phase locked solution $\theta^{*}$ is that $(\omega, \Gamma, \Delta)$ is a solution of $(12 \mathrm{a})$. From Lemma $2,(\omega, \Gamma) \in \mathcal{N}$. Since $(\omega, \Gamma) \in \mathcal{N} \backslash \mathcal{N}_{0}$, Lemma 2 guarantees that the phase differences $\Delta$ of $\theta^{*}$ can locally be uniquely expressed in the form $\Delta=f(\omega, \Gamma)$, for some smooth function $f: \mathbb{R}^{N} \times \mathbb{R}^{N \times N} \rightarrow \mathbb{R}^{N \times N}$. In particular $\Delta$ does not depend on the feedback gain $\gamma$. Consider now the line of $(12 \mathrm{~b})$ relative to the pair of indices $(1,2)$ :

$$
\begin{gathered}
\sum_{i=1}^{N}\left[\gamma_{1 i} \sin \left(\Lambda_{\Omega}(t)+\Delta_{1 i}+2 \theta_{1}^{*}(0)\right)\right. \\
\left.-\gamma_{2 i} \sin \left(\Lambda_{\Omega}(t)+\Delta_{2 i}+2 \theta_{2}^{*}(0)\right)\right]=0, \forall t \geq 0,
\end{gathered}
$$


where $\Lambda_{\Omega}(t):=2 \int_{0}^{t} \Omega(s) d s$, for all $t \geq 0$. Using the identity $\sin (a+b)=\sin a \cos b+\cos a \sin b$ and defining

$$
\begin{aligned}
& \Sigma_{1}:=\sum_{i=1}^{N} \gamma_{1 i} \cos \left(\Delta_{1 i}+2 \theta_{1}^{*}(0)\right)-\gamma_{2 i} \cos \left(\Delta_{2 i}+2 \theta_{2}^{*}(0)\right) \\
& \Sigma_{2}:=\sum_{i=1}^{N} \gamma_{1 i} \sin \left(\Delta_{1 i}+2 \theta_{1}^{*}(0)\right)-\gamma_{2 i} \sin \left(\Delta_{2 i}+2 \theta_{2}^{*}(0)\right)
\end{aligned}
$$

Equation (47) reads $\Sigma_{1} \sin \Lambda_{\Omega}(t)-\Sigma_{2} \cos \Lambda_{\Omega}(t)=0$. Since $\sin \Lambda_{\Omega}(0)=0$ and $\cos \Lambda_{\Omega}(0)=1, \Sigma_{2}$ has to be zero. Moreover, since the phase-locked solution $\theta^{*}$ is oscillating, there exists $t>0$ such that $\sin \Lambda_{\Omega}(t) \neq 0$. Hence, $\Sigma_{1}=0$ as well. Define $b_{1}, b_{2} \in \mathbb{R}^{2 N}$ as

$$
\begin{aligned}
b_{1}(\Delta):= & {\left[\left[\cos \left(\Delta_{1 i}+2 \theta_{1}^{*}(0)\right)\right]_{i=1, \ldots, N}^{T},\right.} \\
& \left.-\left[\cos \left(\Delta_{2 i}+2 \theta_{2}^{*}(0)\right)\right]_{i=1, \ldots, N}^{T}\right]^{T}, \\
b_{2}(\Delta):= & {\left[\left[\sin \left(\Delta_{1 i}+2 \theta_{1}^{*}(0)\right)\right]_{i=1, \ldots, N}^{T},\right.} \\
& \left.-\left[\sin \left(\Delta_{2 i}+2 \theta_{2}^{*}(0)\right)\right]_{i=1, \ldots, N}^{T}\right]^{T} .
\end{aligned}
$$

Note that $b_{1}$ and $b_{2}$ depend only on $\Delta=f(\omega, \Gamma)$ and on the initial conditions. They do not depend on $\gamma$. Hence, by defining $\tilde{\gamma}:=\left(\left[\gamma_{2 i}\right]_{i=1, \ldots, N}^{T},\left[\gamma_{1 i}\right]_{i=1, \ldots, N}^{T}\right)^{T} \in \mathbb{R}^{2 N}$, the condition $\Sigma_{1}=\Sigma_{2}=0$ can be re-written as $\tilde{\gamma}^{T} b_{1}=$ $\tilde{\gamma}^{T} b_{2}=0$ or, equivalently,

$$
\tilde{\gamma} \in b_{1}(f(\omega, \Gamma))^{\perp} \cap b_{2}(f(\omega, \Gamma))^{\perp} .
$$

Let

$$
\mathcal{L}_{0}(\omega, \Gamma):=b_{1}(f(\omega, \Gamma))^{\perp} \cap b_{2}(f(\omega, \Gamma))^{\perp} \subset \mathbb{R}^{2 N} .
$$

Noticing that $b_{1}, b_{2}$ can not be both zero, $\mu\left(\mathcal{L}_{0}(\omega, \Gamma)\right)=$ 0 for all $\omega, \Gamma \in \mathbb{R} \times \mathbb{R}^{N \times N}$. Recalling (50), for all $\omega \in \mathbb{R}^{N} \backslash$ $\mathcal{S}_{0}$, for all $\Gamma \in \mathbb{R}^{N \times N} \backslash \dot{\mathcal{M}}_{0}(\omega)$, and for all $\gamma \in \mathbb{R}^{N \times N} \backslash$ $\mathcal{L}_{0}(\omega, \Gamma)$, system (4) admits no oscillating phase-locked solution, where $\mathcal{M}_{0}, \mathcal{S}_{0}$, and $\mathcal{L}_{0}$ are defined in (45),(46), and (51), respectively, and are all of zero Lebesgue measure. The theorem is proved by noticing that, given $\omega \in$ $\mathbb{R} \backslash \mathcal{S}_{0}$, for any $\Gamma \in \mathbb{R}^{N \times N} \backslash \mathcal{M}_{0}(\omega)$, the set $\left\{\gamma \in \mathbb{R}^{N \times N}\right.$ : $\left.\gamma=\Gamma-k, k \in \mathbb{R}^{N \times N}\right\}=\mathbb{R}^{N \times N}$, that is, given any $\Gamma \in \mathbb{R}^{N \times N} \backslash \mathcal{M}_{0}(\omega), \gamma \notin \mathcal{L}_{0}(\omega, \Gamma)$ for almost all $k$.

\section{Proofs of the Results of Section 4}

\subsection{Proof of Lemma 3}

By the definition (32) of $V(\theta)$, it holds that $\nabla_{\theta} V(\theta)=$ $\left.\nabla_{\theta} V_{I}\right|_{\mathcal{M}}(A \theta)=\left.A^{T} \nabla_{A \theta} V_{I}\right|_{\mathcal{M}}(A \theta)$. Hence

$$
\left.\nabla_{A \theta} V_{I}\right|_{\mathcal{M}}(A \theta)=0 \quad \Rightarrow \quad \nabla_{\theta} V(\theta)=0 .
$$

Since $\operatorname{rank} A=N-1, \operatorname{ker} A^{T}=\{0\}$, it follows that

$$
\nabla_{\theta} V(\theta)=\left.0 \Rightarrow \nabla_{A \theta} V_{I}\right|_{\mathcal{M}}(A \theta)=0,
$$

which proves the first part of the lemma. To prove the second part, we note that if $\theta^{*}$ is a local minimum of $V$ then there exists a neighborhood $U$ of $\theta^{*}$ such that $V(\theta) \geq V\left(\theta^{*}\right)$ for all $\theta \in U$. That is, $\left.V_{I}\right|_{\mathcal{M}}(A \theta) \geq$ $\left.V_{I}\right|_{\mathcal{M}}\left(A \theta^{*}\right)$ for all $\theta \in U$. That is $\left.V_{I}\right|_{\mathcal{M}}(\varphi) \geq\left. V_{I}\right|_{\mathcal{M}}\left(\varphi^{*}\right)$ for all $\varphi \in W=A U$, where $\varphi^{*}=A \theta^{*}$. A similar proof holds for maxima. The third part of the lemma follows from the fact the function $\left.V_{I}\right|_{\mathcal{M}}$ is positive definite and $\left.V_{I}\right|_{\mathcal{M}}(0)=0$, i.e. $\varphi^{*}$ is a local minimum of $\left.V_{I}\right|_{\mathcal{M}}$.

\subsection{Proof of Lemma 4}

From Lemma $3, \varphi^{*} \in \mathcal{M}$ is a critical point of $\left.V_{I}\right|_{\mathcal{M}}$ if and only if $\theta^{*} \in \mathrm{T}^{N}$ is a critical point of $V$, where $\phi^{*}=A \theta^{*}$, and $A$ is defined in (31). Moreover, when $\tilde{\omega}=0,(17)$ can be re-written as

$$
\dot{\theta}_{i}-\dot{\theta}_{j}=K\left(\chi_{j}(\theta)-\chi_{i}(\theta)\right), \quad \forall i, j=1, \ldots, N,
$$

where $\chi(\theta)=\left[\chi_{i}(\theta)\right]_{i=1, \ldots, N}:=\nabla_{\theta} V(\theta)=\sum_{j=1}^{N} E_{i j} \sin \left(\theta_{j}-\right.$ $\left.\theta_{i}\right)$, and $E$ is defined in (25). Hence, $\chi\left(\theta^{*}\right)=\nabla_{\theta} V\left(\theta^{*}\right)=$ 0 if and only if $\phi^{*}=A \theta^{*}$ is a critical point of $\left.V_{I}\right|_{\mathcal{M}}$; and $\chi_{j}\left(\theta^{*}\right)-\chi_{i}\left(\theta^{*}\right)=0$, for all $i, j=1, \ldots, N$, if and only if $B \phi^{*}=B A \theta^{*}$ is a fixed point of the unperturbed incremental dynamics, where $B$ is defined in (29). To prove the lemma it thus suffices to show that

$$
\chi\left(\theta^{*}\right)=0 \quad \Leftrightarrow \quad \chi_{j}\left(\theta^{*}\right)-\chi_{i}\left(\theta^{*}\right)=0, \quad \forall i, j=1, \ldots, N .
$$

One implication is straightforward: if $\chi\left(\theta^{*}\right)=0$, then in particular all of its components are zero, which implies that $\chi_{j}\left(\theta^{*}\right)-\chi_{i}\left(\theta^{*}\right)=0$, for all $i, j=1, \ldots, N$. On the other hand, if $\chi_{j}\left(\theta^{*}\right)-\chi_{i}\left(\theta^{*}\right)=0$ for all $i, j=1, \ldots, N$, then there exists a constant $\bar{\chi}$, such that $\chi_{i}\left(\theta^{*}\right)=\bar{\chi}$ for all $i=1, \ldots, N$. Hence, it results that

$$
N \bar{\chi}=\sum_{i=1}^{N} \chi_{i}\left(\theta^{*}\right)=\sum_{i=1}^{N} \frac{\partial V}{\partial \theta_{i}}\left(\theta^{*}\right)=\sum_{i=1}^{N} \sum_{j=1}^{N} E_{i j} \sin \left(\theta_{j}^{*}-\theta_{i}^{*}\right)
$$

Since the matrix $\tilde{k}$ is symmetric, so is the matrix $E$ (cf. $(25))$, and it results that $\sum_{i=1}^{N} \sum_{j=1}^{N} E_{i j} \sin \left(\theta_{j}^{*}-\theta_{i}^{*}\right)=0$. Consequently, $\chi_{i}\left(\theta^{*}\right)=\bar{\chi}=0$ for all $i=1, \ldots, N$, which proves the converse implication.

\subsection{Proof of Theorem 2}

In order to develop the robustness analysis we consider the Lyapunov function (27), where the incremental variable $\tilde{\theta}$ is defined in (18), and the matrix $E$ is given by (25)-(26). The derivative of $V_{I}$ yields $\dot{V}_{I}(\tilde{\theta})=\left(\nabla_{\tilde{\theta}} V_{I}\right)^{T} \dot{\tilde{\theta}}$, where $\dot{\tilde{\theta}}$ is given by (17). The following claim, whose proof is given in (Franci et al., 2010), provides an alternative expression for $\dot{V}_{I}$. 
Claim 2 If $\tilde{k} \in \mathbb{R}^{N \times N}$ is symmetric, then $\dot{V}_{I}=$ $-2\left(K \chi^{T} \chi+\chi^{T} \tilde{\omega}\right)$, where

$$
\chi(\tilde{\theta}):=\nabla_{\theta} V(\tilde{\theta})=\left[\sum_{j=1}^{N} E_{i j} \sin \left(\theta_{j}-\theta_{i}\right)\right]_{i=1, \ldots, N} .
$$

From Claim 2, it holds that, if $|\chi| \geq \frac{2|\tilde{\omega}|}{K}$, then $\dot{V}_{I} \leq$ $-K \chi^{T} \chi$. However, LISS does not follow yet as these regions are given in terms of $\chi$ rather than $\tilde{\theta}$. In order to estimate these regions in terms of $\tilde{\theta}$, we define $\mathcal{F}$ as the set of critical points of $\left.V_{I}\right|_{\mathcal{M}}$ (i.e. $\mathcal{F}:=\left\{\varphi^{*} \in \mathcal{M}\right.$ : $\left.\left.\left.\nabla_{\varphi} V_{I}\right|_{\mathcal{M}}\left(\varphi^{*}\right)=0\right\}\right)$, where $\mathcal{M}$ and $\left.V_{I}\right|_{\mathcal{M}}$ are defined in (29) and (30), respectively. Then, from Lemma 3 and recalling that $\chi=\nabla_{\theta} V_{I}$, it holds that $|\chi|=0$ if and only if $\tilde{\theta} \in \mathcal{F}$. Since $|\chi|$ is a positive definite function of the distance from $\tilde{\theta}$ to the set $\mathcal{F}$ and is defined on a compact set, (Khalil, 2001, Lemma 4.3) guarantees the existence of a $\mathcal{K}$ function $\sigma$ such that

$$
|\chi| \geq \sigma\left(|\tilde{\theta}|_{\mathcal{F}}\right), \quad \forall \tilde{\theta} \in \mathrm{T}^{(N-1)^{2}} .
$$

The function $\sigma$ can then be taken as $\mathcal{K}_{\infty}$ by choosing a suitable extension outside $T^{(N-1)^{2}}$. Let $\mathcal{U}:=\mathcal{F} \backslash \mathcal{O}_{\tilde{k}}$, where the set $\mathcal{O}_{\tilde{k}}$ is given in Definition 2. In view of Lemma $4, \mathcal{U}$ denotes the set of all the critical points of $\left.V_{I}\right|_{\mathcal{M}}$ which are not asymptotically stable fixed points of the incremental dynamics. Since $\left.\nabla V_{I}\right|_{\mathcal{M}}$ is a Lipschitz function defined on a compact space, it can be different from zero only on a finite collection of open sets. That is, $\mathcal{U}$ and $\mathcal{O}_{\tilde{k}}$ are the finite disjoint unions of closed sets:

$$
\mathcal{U}=\bigcup_{i \in I_{\mathcal{U}}} \nu_{i}, \quad \mathcal{O}_{\tilde{k}}=\bigcup_{i \in I_{\mathcal{O}_{\tilde{k}}}}\left\{\phi_{i}\right\}
$$

where $I_{\mathcal{U}}, I_{\mathcal{O}_{\tilde{k}}} \subset \mathbb{N}$ are finite sets, $\left\{\nu_{i}, i \in I_{\mathcal{U}}\right\}$ is a family of closed subsets of $\mathcal{M}$, and $\left\{\left\{\phi_{i}\right\}, i \in I_{\mathcal{O}_{\tilde{k}}}\right\}$ is a family of singletons of $\mathcal{M}$. We stress that $a \neq b$ implies $a \cap b=\emptyset$ for any $a, b \in\left\{\nu_{i}, i \in I_{\mathcal{U}}\right\} \bigcup\left\{\left\{\phi_{i}\right\}, i \in I_{\mathcal{O}_{\tilde{k}}}\right\}=: \mathcal{F}_{S}$. Define

$$
\delta:=\min _{a, b \in \mathcal{F}_{S}, a \neq b} \inf _{\tilde{\theta} \in a}|\tilde{\theta}|_{b}
$$

which represents the minimum distance between two critical sets, and, at the light of Lemma 4, between two fixed points of the unpertubed incremental dynamics (13). Note that, since $\mathcal{F}_{S}$ is finite, $\delta>0$. Define

$$
\delta_{\omega}^{\prime}=\frac{K}{2} \sigma\left(\frac{\delta}{2}\right), \quad \delta_{\tilde{\theta}}:=\frac{\delta}{2}
$$

$\delta_{\tilde{\theta}}$ then gives an estimate of the radius of attraction, modulo the shape of the level sets of $V_{I}$. The following claim is proven in (Franci et al., 2010).
Claim 3 For all $i \in I_{\mathcal{O}_{\tilde{k}}}, \tilde{\theta} \in \mathcal{B}\left(\phi_{i}, \delta_{\tilde{\theta}}\right)$, and $|\tilde{\omega}| \leq \delta_{\omega}^{\prime}$,

$$
\left|\tilde{\theta}-\phi_{i}\right| \geq \sigma^{-1}\left(\frac{2|\tilde{\omega}|}{K}\right) \Rightarrow \dot{V}_{I} \leq-K \sigma^{2}\left(\left|\tilde{\theta}-\phi_{i}\right|\right) .
$$

For all $i \in I_{\mathcal{O}_{\tilde{k}}}$, the function $V_{I}(\tilde{\theta})-V_{I}\left(\phi_{i}\right)$ is zero for $\tilde{\theta}=\phi_{i}$, and strictly positive for all $\theta \in \mathcal{B}\left(\phi_{i}, \delta_{\tilde{\theta}}\right) \backslash\left\{\phi_{i}\right\}$. Noticing that $\mathcal{B}\left(\phi_{i}, \delta_{\tilde{\theta}}\right)$ is compact, (Khalil, 2001, Lemma 4.3) guarantees the existence of $\mathcal{K}$ functions $\underline{\alpha}_{i}, \bar{\alpha}_{i}$ defined on $\left[0, \delta_{\tilde{\theta}}\right]$ such that, for all $\tilde{\theta} \in \mathcal{B}\left(\phi_{i}, \delta_{\tilde{\theta}}\right)$,

$$
\underline{\alpha}_{i}\left(\left|\tilde{\theta}-\phi_{i}\right|\right) \leq V_{I}(\tilde{\theta})-V_{I}\left(\phi_{i}\right) \leq \bar{\alpha}_{i}\left(\left|\tilde{\theta}-\phi_{i}\right|\right) .
$$

The two functions can then be picked as $\mathcal{K}_{\infty}$ by choosing a suitable prolongation on $\mathbb{R}_{\geq 0}$. Define the $\mathcal{K}_{\infty}$ functions

$$
\underline{\alpha}(s):=\min _{i \in I_{\mathcal{O}_{\tilde{k}}}} \underline{\alpha}_{i}(s), \bar{\alpha}(s):=\max _{i \in I_{\mathcal{O}_{\tilde{k}}}} \bar{\alpha}_{i}(s), \forall s \geq 0 .
$$

It then holds that, for all $i \in I_{\mathcal{O}_{\tilde{k}}}$ and all $\tilde{\theta} \in \mathcal{B}\left(\phi_{i}, \delta_{\tilde{\theta}}\right)$,

$$
\underline{\alpha}\left(\left|\tilde{\theta}-\phi_{i}\right|\right) \leq V_{I}(\tilde{\theta})-V_{I}\left(\phi_{i}\right) \leq \bar{\alpha}\left(\left|\tilde{\theta}-\phi_{i}\right|\right) .
$$

In view of Claim 3 and (58), it follows from (Isidori, 1999, Remark 10.4.3) that an estimate of the ISS gain and on the tolerated input bound are given by

$$
\begin{aligned}
\rho(s) & :=\underline{\alpha}^{-1} \circ \bar{\alpha} \circ \sigma^{-1}\left(\frac{2}{K} s\right), \forall s \geq 0 \\
\delta_{\omega} & :=\rho^{-1}\left(\delta_{\tilde{\theta}}\right) \leq \delta_{\omega}^{\prime},
\end{aligned}
$$

where $\sigma$ is defined in (52). From (Isidori, 1999, Section 10.4) and Claim 3, it follows that, for all $\|\tilde{\omega}\| \leq \delta_{\omega}$, the set $\mathcal{B}\left(\mathcal{O}_{\tilde{k}}, \delta_{\tilde{\theta}}\right)$ is forward invariant for the system (17). Furthermore, invoking (Sontag and Wang, 1996) and (Isidori, 1999, Section 10.4), Claim 3 thus implies LISS with respect to inputs satisfying $\|\tilde{\omega}\| \leq \delta_{\omega}$, meaning that there exists a class $\mathcal{K} \mathcal{L}$ function $\beta$ such that, for all $\|\tilde{\omega}\| \leq \delta_{\omega}$, and all $\tilde{\theta}_{0} \in \mathcal{B}\left(\mathcal{O}_{\tilde{k}}, \delta_{\tilde{\theta}}\right)$, the trajectory of (17) satisfies $|\tilde{\theta}(t)| \leq \beta\left(\left|\tilde{\theta}_{0}\right|, t\right)+\rho(\|\tilde{\omega}\|)$, for all $t \geq 0$.

\subsection{Proof of Corollary 1}

The Corollary is a trivial consequence of Theorem 2 by noting that, since $\left|\left(\tilde{I}_{M F}\right)_{i}\right|=\left|\left(I_{M F}\right)_{i}-\frac{1}{N} \sum_{j}\left(I_{M F}\right)_{j}\right| \leq$ $\max _{j}\left|\left(I_{M F}\right)_{j}\right|$ and $\left|\left(I_{M F}\right)_{i}\right|<2 N \bar{\gamma}$, for all $i=1, \ldots, N$, it results that $\left|\tilde{I}_{M F}\right|<2 \bar{\gamma} \sqrt{N} N$. By letting $\delta_{\omega}$ be defined as in (60), from Theorem 2, the system is LISS, provided that $\left|\omega^{\perp}\right|+\left|\tilde{I}_{M F}\right|<\left|\omega^{\perp}\right|+2 \bar{\gamma} \sqrt{N} N \leq \delta_{\omega}$. 


\subsection{Proof of Proposition 1}

\section{Input-to-State Gain}

We start by computing the ISS gain $\rho$, defined in (59), in the particular case of all-to-all coupling and show that it can be taken as a linear function. The first step is to compute the function $\sigma$, defined in (52), with respect to the origin of the incremental dynamics. That is we have to find a class $\mathcal{K}_{\infty}$ function $\sigma$, such that $|\chi(\tilde{\theta})| \geq$ $\sigma(|\tilde{\theta}|)$ for all $\tilde{\theta}$ in some neighborhood of the origin of the incremental dynamics, where $\chi$ is defined in Claim 2. The following claim, whose proof is given in Section 7, gives an explicit expression of this function on the set $\mathcal{D}_{0}=\left\{\tilde{\theta} \in \mathrm{T}^{(N-1)^{2}}:|\tilde{\theta}|_{\infty} \leq \frac{\pi}{2}\right\}$, as defined in the statement of Proposition 1.

Claim 4 In the case of all-to-all coupling, the function $\chi$ defined in Claim 2 satisfies, for any $\tilde{\theta} \in \mathcal{D}_{0},|\chi(\tilde{\theta})| \geq \frac{|\tilde{\theta}|}{\pi}$, that is the function $\sigma$ in (52) can be picked as $\sigma(r)=\frac{r}{\pi}$, for all $r \geq 0$.

At the light of Claim 4, the ISS gain $\rho$ can be easily computed through (59). Indeed, in the all-to-all case, the entries of the matrix $E$, introduced in (25), are all equal to 1 , and the Lyapunov function $V_{I}$, provided in (27), thus becomes

$$
V_{I}(\tilde{\theta})=2 \sum_{i, j=1}^{N} \sin ^{2}\left(\frac{\theta_{i}-\theta_{j}}{2}\right)
$$

Using the fact that $z \geq \sin z \geq \frac{2}{\pi} z$, for all $0 \leq z \leq \frac{\pi}{2}$, it follows that, for all $\tilde{\theta} \in \mathcal{D}_{0}$,

$$
\frac{2}{\pi^{2}}|\tilde{\theta}|^{2} \leq V_{I}(\tilde{\theta}) \leq \frac{1}{2}|\tilde{\theta}|^{2}
$$

Recalling the definition of the upper $\bar{\alpha}$ and lower $\underline{\alpha}$ estimates of the Lyapunov function with respect the set of asymptotically stable fixed point (57), and that, in the all-to-all case, this set reduces to the origin, we conclude that

$$
\underline{\alpha}(r)=\frac{2}{\pi^{2}} r^{2}, \quad \bar{\alpha}(r)=\frac{1}{2} r^{2}, \forall r \geq 0 .
$$

In view of Claim 4 and (59), it follows that the ISS gain $\rho$ in the statement of Theorem 2, can be chose as

$$
\rho(r)=\frac{\pi^{2}}{K} r
$$

\section{Input bound and invariant set}

For Claim 4, the ISS gain computed in the previous section is valid as soon as $\tilde{\theta}$ belongs to $\mathcal{D}_{0}$. In the following we compute an input bound which guarantees that trajectories starting in $\mathcal{D}_{0}$ remain inside $\mathcal{D}_{0}$. For the sake of generality, we actually show the forward invariance of $\mathcal{D}_{\epsilon}$ for any $\epsilon \in\left[0, \frac{\pi}{2}\right]$. To that end, we start by the following technical claim, whose proof is given in Section 7 .

Claim 5 Given any $0 \leq \delta \leq \pi$, the following holds true:

$$
|\tilde{\theta}| \leq \sqrt{N} \delta \Rightarrow \max _{i, j=1, \ldots, N}\left|\theta_{i}-\theta_{j}\right| \leq \delta
$$

At the light of Claim 5, and in view of (19) and (62), we can compute the input bound $\delta_{\omega}^{\epsilon}$ which lets $\mathcal{D}_{\epsilon}$ be invariant for the systems (17) by imposing $\rho\left(\delta_{\omega}^{\epsilon}\right)=\sqrt{N}\left(\frac{\pi}{2}-\epsilon\right)$, where $\rho(s)=\frac{\pi^{2}}{K} s$ is the ISS gain in the statement of Theorem 2. This gives

$$
\delta_{\omega}^{\epsilon}=\frac{K \sqrt{N}}{\pi^{2}}\left(\frac{\pi}{2}-\epsilon\right) .
$$

\section{Exponential convergence and attractivity of $\mathcal{D}_{\epsilon}$}

From Claims 3 and 4, and (61), it holds that, for all $|\tilde{\omega}| \leq \delta_{\omega}^{\epsilon}$, and all $\tilde{\theta} \in \mathcal{D}_{0}$,

$$
|\tilde{\theta}| \geq \frac{2 \pi}{K}|\tilde{\omega}| \Rightarrow \dot{V}_{I} \leq-\frac{K}{\pi^{2}}|\tilde{\theta}|^{2} \leq-\frac{2 K}{\pi^{2}} V_{I} .
$$

Invoking the comparison Lemma (Khalil, 2001, Lemma 3.4 ), it follows that, for all $t \geq 0$,

$$
\min _{0 \leq s \leq t}|\tilde{\theta}(s)| \geq \frac{2 \pi}{K}\|\tilde{\omega}\| \Rightarrow V_{I}(\tilde{\theta}(t)) \leq V_{I}(\tilde{\theta}(0)) e^{-\frac{2 K}{\pi^{2}} t}
$$

From (61), this also implies that, for all $t \geq 0$

$$
\min _{0 \leq s \leq t}|\tilde{\theta}(s)| \geq \frac{2 \pi}{K}\|\tilde{\omega}\| \Rightarrow|\tilde{\theta}(t)| \leq \frac{\pi}{2}|\tilde{\theta}(0)| e^{-\frac{K}{\pi^{2}} t}
$$

Recalling the explicit expression of ISS gain (62), this implies that the system is exponentially input-to-state stable (see for instance (Isidori, 1999, Section 10.4) and (Khalil, 2001, Lemma 4.4 and Theorem 4.10)), and in particular that for all $\|\tilde{\omega}\| \leq \delta_{\omega}^{\epsilon}$ and all $\tilde{\theta}_{0}$ in $\mathcal{D}_{0}$,

$$
|\tilde{\theta}(t)| \leq \frac{\pi}{2}\left|\tilde{\theta}_{0}\right| e^{-\frac{K}{\pi^{2}} t}+\frac{\pi^{2}}{K}\|\tilde{\omega}\|, \forall t \geq 0
$$

Noticing finally that, if $\|\tilde{\omega}\| \leq \delta_{\omega}^{\epsilon}$, (63) guarantees that

$$
|\tilde{\theta}(t)| \leq \frac{\pi}{2}\left|\tilde{\theta}_{0}\right| e^{-\frac{K}{\pi^{2}} t}+\sqrt{N}\left(\frac{\pi}{2}-\epsilon\right), \forall t \geq 0,
$$

Claim 5 implies the attractivity of $\mathcal{D}_{\epsilon}$ for all $\|\tilde{\omega}\| \leq \delta_{\omega}^{\epsilon}$. 


\section{Technical proofs}

\subsection{Proof of Claim 1}

We start by introducing the notation that will be used along the proof. The two sets $\mathcal{E}$ and $\mathcal{G}$ are defined as follows $\mathcal{E}:=\mathbb{R}^{N \times N} \times \mathbb{R}^{N-1}$ and $\mathcal{G}:=\mathbb{R}^{N-1} \times \mathbb{R}^{N \times N}$. Unless differently specified, if $A \subset \mathbb{R}^{n}, \mu(A)$ denotes the Lebesgue measure of $A$ in $\mathbb{R}^{n}$.

Given $\omega \in \mathbb{R}^{N}$, let $\hat{\omega}_{i}:=\omega_{i}-\omega_{N}, i=1, \ldots, N-1$, and

$$
\hat{\omega}:=\left[\hat{\omega}_{i}\right]_{i=1, \ldots, N-1} .
$$

The set $\mathcal{N}$ and $\mathcal{N}_{0}$, defined, respectively, in (43) and (44), can be characterized in terms of $\hat{\omega}$ as follows. Let, for all $\omega \in \mathbb{R}^{N}$, all $\Gamma \in \mathbb{R}^{N \times N}$, and all $y \in \mathbb{R}^{N-1}$,

$$
\begin{aligned}
& \hat{F}_{i}\left(\hat{\omega}_{i}, \Gamma, y\right):= \sum_{\substack{k=1\\
}}^{N-1}\left[\Gamma_{i k} \sin \left(y_{k}-y_{i}\right)-\Gamma_{N k} \sin y_{k}\right]- \\
& \sin y_{i}, \quad i=1, \ldots, N-1,
\end{aligned}
$$

and define

$$
\hat{F}(\hat{\omega}, \Gamma, y):=\left[\hat{F}_{i}(\hat{\omega}, \Gamma, y)\right]_{i=1, \ldots, N} .
$$

Moreover, let the set of all pairs $(\hat{\omega}, \Gamma)$ that admit a solution $y$ to $\hat{F}(\hat{\omega}, \Gamma, y)=0$ be denoted as

$$
\Sigma:=\left\{(\hat{\omega}, \Gamma) \in \mathcal{G}: \exists y \in \mathbb{R}^{N-1}: \hat{F}(\hat{\omega}, \Gamma, y)=0\right\}
$$

and let the subset $\Sigma_{0} \subset \Sigma$ be defined as

$$
\begin{aligned}
\Sigma_{0}:=\{(\hat{\omega}, \Gamma) & \in \mathcal{G}: \exists y \in \mathbb{R}^{N-1}: \\
\hat{F}(\hat{\omega}, \Gamma, y) & =0, \Gamma \in S(y)\},
\end{aligned}
$$

where, for each $y \in \mathbb{R}^{N-1}$, the set $S(y)$ is defined in (42). We are going to show that the sets $\Sigma$ and $\Sigma_{0}$ completely characterize, respectively, the sets $\mathcal{N}$ and $\mathcal{N}_{0}$.

STEP 1:We claim that the set $\mathcal{N}$ and $\mathcal{N}_{0}$, defined, respectively, by (43) and (44), are the images of, respectively, $\Sigma \times \mathbb{R}$ and $\Sigma_{0} \times \mathbb{R}$ by a linear map of determinant 1 . In particular, if $\mu\left(\Sigma_{0}\right)=0$, then $\mu\left(\mathcal{N}_{0}\right)=0$.

Given $\hat{\omega} \in \mathbb{R}^{N-1}$, let

$$
\begin{aligned}
& I_{\hat{\omega}}:= \\
& \left\{\omega \in \mathbb{R}^{N}: \omega_{i}=\hat{\omega}_{i}+\omega_{N} \cdot i=1, \ldots, N-1, \omega_{N} \in \mathbb{R}\right\} .
\end{aligned}
$$

Let the linear map $M: \mathbb{R}^{N} \rightarrow \mathbb{R}^{N}$ be defined as

$$
M x:=\left[I_{N}-\left[\begin{array}{c}
1_{N-1} \\
0
\end{array}\right][0, \ldots, 0,1]\right] x, \quad \forall x \in \mathbb{R}^{N} .
$$

Note that $M$ has determinant 1 . The set $\hat{\omega} \times \mathbb{R}$ is the image of the set $I_{\hat{\omega}}$ through the linear invertible map $M$, that is

$$
\hat{\omega} \times \mathbb{R}=M\left(I_{\hat{\omega}}\right) .
$$

By definitions (39),(64),(65),(68), it follows that $\hat{F}(\hat{\omega}, \Gamma, y)=F(\omega, \Gamma, y)$, for all $\omega \in I_{\hat{\omega}}$. Hence,

$$
F(\omega, \Gamma, y)=0 \Rightarrow \hat{F}(\hat{\omega}, \Gamma, y)=0
$$

and

$$
\hat{F}(\hat{\omega}, \Gamma, y)=0 \Rightarrow F(\omega, \Gamma, y)=0, \forall \omega \in I_{\hat{\omega}}
$$

Relation (71) implies that, if $\hat{\omega} \in \Sigma$, then $I_{\hat{\omega}} \subset \mathcal{N}$. Since, from $(69), I_{\hat{\omega}}=M^{-1}(\hat{\omega} \times \mathbb{R})$ this also implies that, for all $\hat{\omega} \in \Sigma, M^{-1}(\hat{\omega} \times \mathbb{R}) \subset \mathcal{N}$, which shows that

$$
M^{-1}(\Sigma \times \mathbb{R}) \subset \mathcal{N}
$$

The converse inclusion follows by noticing that, given $\omega \in \mathcal{N},(70)$ implies that $\hat{\omega} \in \Sigma$. It follows that, given $\omega \in \mathcal{N}, \hat{\omega} \times \mathbb{R} \subset \Sigma \times \mathbb{R}$, that is, from $(69), M\left(I_{\hat{\omega}}\right) \subset \Sigma \times \mathbb{R}$. Since, by construction, $\omega \in I_{\hat{\omega}}$, we conclude that, for all $\omega \in \mathcal{N}, M(\omega) \in \Sigma \times \mathbb{R}$, that is

$$
M(\mathcal{N}) \subset \Sigma \times \mathbb{R}
$$

which shows the first part of STEP 1 for $\mathcal{N}$. The statement for $\mathcal{N}_{0}$ follows similarly.

To show that $\mu\left(\mathcal{N}_{0}\right)=0$ whenever $\mu\left(\Sigma_{0}\right)=0$, recall that the Lebesgue measure is invariant under linear maps of determinant 1 and note that, given a collection of open intervals $\left\{I_{i}\right\}_{i \in \mathbb{N}}$, such that $\bigcup_{i \in \mathbb{N}} I_{i}=\mathbb{R}, \Sigma_{0} \times \mathbb{R}$ can be written as the countable union $\Sigma_{0} \times \mathbb{R}=\bigcup_{i \in \mathbb{N}} \Sigma_{0} \times I_{i}$. Thus, since, for all $i \in \mathbb{N}, \mu\left(\Sigma_{0} \times I_{i}\right)=\mu\left(\Sigma_{0}\right) \mu\left(I_{i}\right)$ (see for instance (Billingsley, 1995, Section 12)), if $\mu\left(\Sigma_{0}\right)=0$, then $\mu\left(\Sigma_{0} \times I_{i}\right)=0$, for all $i \in \mathbb{N}$. That is, if $\mu\left(\Sigma_{0}\right)=0$, then $\mathcal{N}_{0}$ is the image through an invertible linear map of the union of a countable collection of sets of zero Lebesgue measure, and, hence, of zero Lebesgue measure, which shows STEP 1.

Before introducing the second step of the proof we need the following notation. Let $\mathcal{E}_{0}$ be the set of matrices $\Gamma$ and phase-differences $y$ such that the Jacobian of $F$ is singular, that is

$$
\mathcal{E}_{0}:=\{(\Gamma, y) \in \mathcal{E}: \Gamma \in S(y)\}
$$

where, for all $y \in \mathbb{R}^{N-1}$, the set $S(y)$ is defined in (42).

Let $\mathcal{F}$, be the set of triplet $(\hat{\omega}, \Gamma, y)$ that are solution to $F=0$, that is

$$
\mathcal{F}:=\left\{(\hat{\omega}, \Gamma, y) \in \mathcal{G} \times \mathbb{R}^{N-1}: \hat{F}(\hat{\omega}, \Gamma, y)=0\right\}
$$


and $\mathcal{F}_{0}$ the set of triplet $(\hat{\omega}, \Gamma, y)$ that are solution to $F=0$, and, moreover, such that the Jacobian of $F$ is singular, that is

$\mathcal{F}_{0}:=\left\{(\hat{\omega}, \Gamma, y) \in \mathcal{G} \times \mathbb{R}^{N-1}: \hat{F}(\hat{\omega}, \Gamma, y)=0, \Gamma \in S(y)\right\}$,

The next step shows that $\Sigma$ is the image of $\mathcal{E}$ through a smooth mapping $T$, and that $\Sigma_{0}$ is the image of $\mathcal{E}_{0}$ through $T$. This will allow to study the measure of $\Sigma_{0}$ as a function of the measure of $\mathcal{E}_{0}$.

STEP 2: There exists a smooth map $T: \mathcal{E} \rightarrow \mathcal{G}$, such that $\Sigma=T(\mathcal{E})$, and $\Sigma_{0}=T\left(\mathcal{E}_{0}\right)$.

Consider the set $\mathcal{F}$ defined in (73). Since $\frac{\partial \hat{F}_{i}}{\partial \hat{\omega}_{j}}=\delta_{i j}$, where $\delta_{i j}$ denotes the Kroenecker symbol, it follows from (Lee, 2006, Theorem 8.8) that $\mathcal{F}$ is an embedded submanifold of codimension $N-1$. In particular $\mathcal{F}$ can be described as the graph of the smooth function

$$
g:=-F(0, \cdot, \cdot): \mathcal{E} \rightarrow \mathbb{R}^{N-1},
$$

as

$$
\mathcal{F}=\left\{(\hat{\omega}, \Gamma, y) \in \mathcal{G} \times \mathbb{R}^{N-1}: \hat{\omega}=g(\Gamma, y)\right\}
$$

Similarly $\mathcal{F}_{0}=\left\{(\hat{\omega}, \Gamma, y) \in \mathcal{G} \times \mathbb{R}^{N-1}:(\Gamma, y) \in \mathcal{E}_{0}, \hat{\omega}=\right.$ $g(\Gamma, y)\}$. Hence, the point of $\mathcal{F}$ are the image of the point of $\mathcal{E}$ through the smooth mapping (lift) $L: \mathcal{E} \rightarrow$ $\mathcal{G} \times \mathcal{R}^{N-1}$, defined by

$$
L(\Gamma, y):=(g(\Gamma, y), \Gamma, y) .
$$

Moreover, it follows directly from the definition of $\Sigma$ that $\Sigma=P_{\mathcal{G}} \mathcal{F}$ and $\Sigma_{0}=P_{\mathcal{G}} \mathcal{F}_{0}$, where $P_{\mathcal{G}}$ is the projection of $\mathcal{G} \times \mathbb{R}^{N-1}$ on $\mathcal{G}$, defined by $P_{\mathcal{G}}(\hat{\omega}, \Gamma, y)=(\hat{\omega}, \Gamma)$.

Hence, by defining $T:=P_{\mathcal{G}} \circ L: \mathcal{E} \rightarrow \mathcal{G}$, as

$$
T(\Gamma, y)=(g(\Gamma, y), \Gamma), \forall(\Gamma, y) \in \mathcal{E}
$$

(see fig. also 4 ), we conclude that, $\Sigma=T(\mathcal{E})$, and $\Sigma_{0}=$ $T\left(\mathcal{E}_{0}\right)$.

STEP 3: The Lebesgue measure of $\Sigma_{0}$ is zero.

Since $\mathcal{E}_{0}$ is given by the zeros of an analytic function, $\mu\left(\mathcal{E}_{0}\right)=0$. Since $\Sigma_{0}$ is the image through a smooth map $T: \mathcal{E} \rightarrow \mathcal{G}$ of a set of zero measure (cf. STEP 2), $\Sigma_{0}$ has zero measure (Lee, 2006, Lemma 10.2).

The statement of the claim follows directly from STEP 1 and STEP 3

\subsection{Proof of Claim 2}

Consider the derivative of the incremental Lyapunov function $V_{I}$, defined in (27), along the trajectories of the

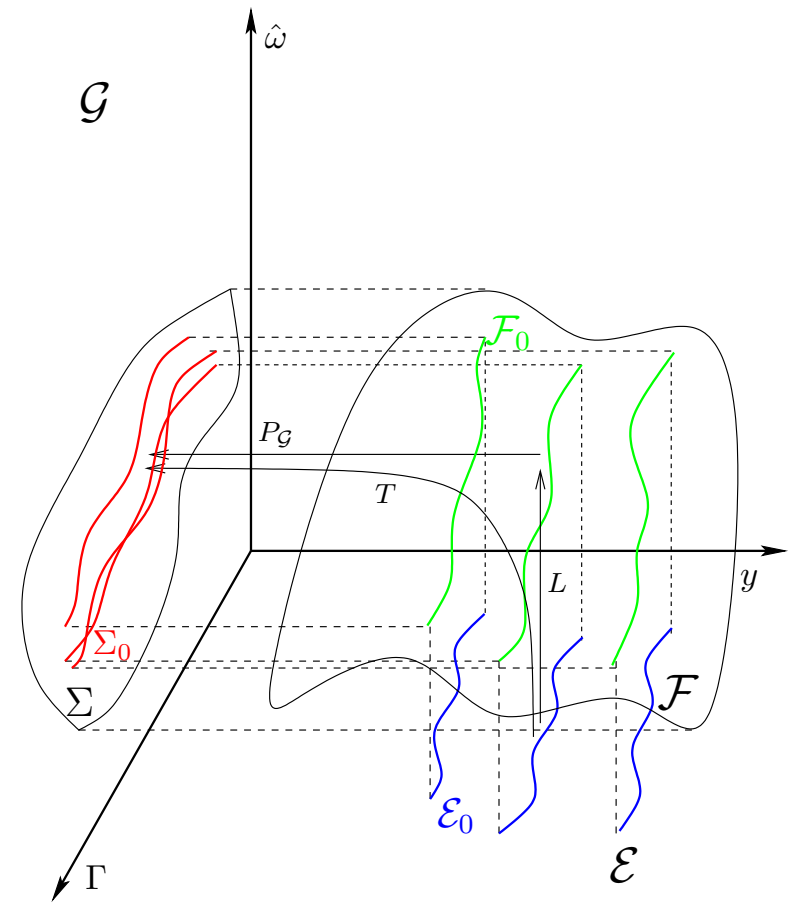

Fig. 4. Construction of the smooth map $T: \mathcal{E} \rightarrow \mathcal{G}$. incremental dynamics (17):

$$
\begin{aligned}
\dot{V}_{I}(\tilde{\theta}):=\left(\nabla_{\tilde{\theta}} V_{I}\right)^{T} \dot{\tilde{\theta}} & =\sum_{i, j=1}^{N} E_{i j} \sin \left(\theta_{j}-\theta_{i}\right)\left(\dot{\theta}_{j}-\dot{\theta}_{i}\right) \\
& =-2 \sum_{i, j=1}^{N} E_{i j} \sin \left(\theta_{j}-\theta_{i}\right) \dot{\theta}_{i}
\end{aligned}
$$

where the last equality comes from the fact that, since $E$ is a symmetric matrix, $\sum_{i, j=1}^{N} E_{i j} \sin \left(\theta_{j}-\theta_{i}\right) \dot{\theta}_{j}=$ $-\sum_{i, j=1}^{N} E_{i j} \sin \left(\theta_{j}-\theta_{i}\right) \dot{\theta}_{i}$. For the same reason it holds that $\bar{\omega} \sum_{i, j=1}^{N} E_{i j} \sin \left(\theta_{j}-\theta_{i}\right)=0$. Since, from (13), $\dot{\theta}_{i}=$ $\bar{\omega}+\tilde{\omega}_{i}+K \sum_{l=1}^{N} E_{i l} \sin \left(\theta_{l}-\theta_{i}\right)$, we get that

$$
\begin{aligned}
\dot{V}_{I} & =-2 \sum_{i=1}^{N}\left(\sum_{j=1}^{N} E_{i j} \sin \left(\theta_{j}-\theta_{i}\right)\right)\left(K \sum_{l=1}^{N} E_{i l} \sin \left(\theta_{l}-\theta_{i}\right)+\tilde{\omega}_{i}\right) \\
& =-2\left(K \nabla V^{T} \nabla V+\nabla V^{T} \tilde{\omega}\right)
\end{aligned}
$$

which proves the claim.

\subsection{Proof of Claim 3}

From Claim 2 it holds that $\dot{V}_{I}=-2 K|\chi|^{2}-2 \chi^{T} \tilde{\omega} \leq$ $-2 K|\chi|^{2}+2|\chi||\tilde{\omega}|$. That is

$$
|\chi| \geq \frac{2|\tilde{\omega}|}{K}, \quad \Rightarrow \quad \dot{V}_{I} \leq-K|\chi|^{2}
$$


In view of (??)-(55), $|\tilde{\omega}| \leq \delta_{\omega}^{\prime}$ implies that $\sigma^{-1}\left(\frac{2|\tilde{\omega}|}{K}\right) \leq$ $\delta_{\tilde{\theta}}$. Recalling that, for all $\tilde{\theta} \in \mathcal{B}\left(\phi_{i}, \delta_{\tilde{\theta}}\right),|\tilde{\theta}|_{\mathcal{F}}=\left|\tilde{\theta}-\phi_{i}\right|$, it results that

$$
\left|\tilde{\theta}-\phi_{i}\right| \geq \sigma^{-1}\left(\frac{2|\tilde{\omega}|}{K}\right) \Rightarrow|\chi| \geq \frac{2|\tilde{\omega}|}{K} .
$$

Since (52) ensures that $-|\chi|^{2} \leq-\sigma^{2}\left(\left|\tilde{\theta}-\phi_{i}\right|\right)$, at the light of (75) and (76), it results that

$$
\left|\tilde{\theta}-\phi_{i}\right| \geq \sigma^{-1}\left(\frac{2|\tilde{\omega}|}{K}\right) \Rightarrow \dot{V}_{I} \leq-K \sigma^{2}\left(\left|\tilde{\theta}-\phi_{i}\right|\right) .
$$

\subsection{Proof of Claim 4}

In the case of all-to-all coupling, the vector $\chi$ defined in Claim 2 reads

$$
\chi(\tilde{\theta})=\left[\sum_{j=1}^{N} \sin \left(\theta_{j}-\theta_{i}\right)\right]_{i=1, \ldots, N} .
$$

Therefore, the norm inequality $|\tilde{\theta}|_{2} \geq|\tilde{\theta}|_{\infty}$ implies

$$
|\chi(\tilde{\theta})|_{2} \geq \max _{i=1, \ldots, N}\left|\sum_{j=1}^{N} \sin \left(\theta_{j}-\theta_{i}\right)\right| .
$$

Now, since $\tilde{\theta} \in \mathcal{D}_{0}$, we have $\left|\theta_{i}-\theta_{j}\right| \leq \frac{\pi}{2}$, which implies that the phases of all oscillators belong to the same quarter of circle. We can thus renumber the indexes of the oscillator phases in such a way that $\theta_{i} \leq \theta_{j}$ whenever $i<j$. First step - For a given $\tilde{\theta}$, in order to find a tight lower bound on $|\chi(\tilde{\theta})|_{2}$, we are going to show that

$$
\begin{gathered}
\max _{i=1, \ldots, N}\left|\sum_{j=1}^{N} \sin \left(\theta_{j}-\theta_{i}\right)\right| \\
=\max \left\{\sum_{j=1}^{N} \sin \left|\theta_{j}-\theta_{1}\right|, \sum_{j=1}^{N} \sin \left|\theta_{j}-\theta_{N}\right|\right\} .
\end{gathered}
$$

On the one hand, for all $j=1, \ldots, N$, we have $0 \leq$ $\theta_{j}-\theta_{1} \leq \frac{\pi}{2}$ and $0 \leq \theta_{N}-\theta_{j} \leq \frac{\pi}{2}$. It follows that

$$
\left|\sum_{j=1}^{N} \sin \left(\theta_{j}-\theta_{1}\right)\right|=\sum_{j=1}^{N} \sin \left|\theta_{j}-\theta_{1}\right|
$$

and

$$
\left|\sum_{j=1}^{N} \sin \left(\theta_{j}-\theta_{N}\right)\right|=\sum_{j=1}^{N} \sin \left|\theta_{j}-\theta_{N}\right|
$$

On the other hand, for any $i \notin\{1, N\}$, we have that, for any $j<i, 0 \leq \theta_{i}-\theta_{j} \leq \frac{\pi}{2}$; while, for any $j>i$, $0 \leq \theta_{j}-\theta_{i} \leq \frac{\pi}{2}$. That is

$$
\left|\sum_{j=1}^{N} \sin \left(\theta_{j}-\theta_{i}\right)\right|=\left|\sum_{j=i+1}^{N} \sin \right| \theta_{j}-\theta_{i}\left|-\sum_{j=1}^{i-1} \sin \right| \theta_{j}-\theta_{i}||
$$

Now, for all $\tilde{\theta} \in \mathcal{D}_{0}$, if $i>j$, then $\left|\theta_{j}-\theta_{i}\right| \leq\left|\theta_{j}-\theta_{N}\right|$, while, if $i<j$, then $\left|\theta_{j}-\theta_{i}\right| \leq\left|\theta_{j}-\theta_{1}\right|$. Hence, for all $\tilde{\theta} \in \mathcal{D}_{0}$

$$
\sum_{j=1}^{i-1} \sin \left|\theta_{j}-\theta_{i}\right| \leq \sum_{j=1}^{N} \sin \left|\theta_{j}-\theta_{N}\right|, \quad \forall i \notin\{1, N\}
$$

and

$$
\sum_{j=i+1}^{N} \sin \left|\theta_{j}-\theta_{i}\right| \leq \sum_{j=1}^{N} \sin \left|\theta_{j}-\theta_{1}\right|, \quad \forall i \notin\{1, N\}
$$

Recalling that, for all $a, b \geq 0,|a-b| \leq \max \{a, b\}$, it then follows that, for any $i \notin\{1, N\}$,

$$
\begin{aligned}
& \leq \max \left\{\sum_{j=1}^{N} \sin \left(\theta_{j}-\theta_{i}\right) \mid\right. \\
& \left.\leq \max \left|\theta_{j}-\theta_{i}\right|, \sum_{j=1}^{N} \sin \left|\theta_{j}-\theta_{i}\right|\right\} \\
& \left\{\sum_{j=1}^{N} \sin \left|\theta_{j}-\theta_{1}\right|, \sum_{j=1}^{N} \sin \left|\theta_{j}-\theta_{N}\right|\right\} .
\end{aligned}
$$

Therefore, combining (78), (79), and (80), we obtain (77), which ends the first step of the proof. Second step - Using the fact that $\sin z \geq \frac{2}{\pi} z$, for all $z \in\left[0, \frac{\pi}{2}\right]$, Equation (77) yields

$$
\begin{aligned}
|\chi(\tilde{\theta})|_{2} & \geq \max \left\{\sum_{j=1}^{N} \sin \left|\theta_{j}-\theta_{1}\right|, \sum_{j=1}^{N} \sin \left|\theta_{j}-\theta_{N}\right|\right\} \\
& \geq \frac{2}{\pi} \max \left\{\sum_{j=1}^{N}\left|\theta_{j}-\theta_{1}\right|, \sum_{j=1}^{N}\left|\theta_{j}-\theta_{N}\right|\right\} \\
& \geq \frac{2}{\pi} \max \left\{\sum_{j=1}^{N}\left(\theta_{j}-\theta_{1}\right), \sum_{j=1}^{N}\left(\theta_{N}-\theta_{j}\right)\right\} .
\end{aligned}
$$


Or, equivalently, by defining $\delta:=\theta_{N}-\theta_{1}$, with $0 \leq \delta \leq$ $\frac{\pi}{2}$

$$
|\chi(\tilde{\theta})|_{2} \geq \frac{2}{\pi} \max \left\{\sum_{j=1}^{N}\left(\theta_{j}-\theta_{1}\right), \sum_{j=1}^{N}\left[\delta-\left(\theta_{j}-\theta_{1}\right)\right]\right\} .
$$

For notation purposes, define $I_{\tilde{\theta}}:=[0, \delta]^{N-2}, x_{i}:=$ $\theta_{i+1}-\theta_{1}$, for all $i=1, \ldots, N-2$, and $x:=\left[x_{i}\right]_{i=1, \ldots, N-2} \in$ $I_{\tilde{\theta}}$. Then

$$
\begin{aligned}
& \max \left\{\sum_{j=1}^{N}\left(\theta_{j}-\theta_{1}\right), \sum_{j=1}^{N}\left[\delta-\left(\theta_{j}-\theta_{1}\right)\right]\right\} \\
& =\max \left\{\delta+\sum_{i=1}^{N-2} x_{i}, \delta+\sum_{i=1}^{N-2}\left(\delta-x_{i}\right)\right\} \\
& =\delta+\max \left\{\sum_{i=1}^{N-2} x_{i}, \sum_{i=1}^{N-2}\left(\delta-x_{i}\right)\right\} .
\end{aligned}
$$

In order to obtain the desired bound, we minimize the function $f: I_{\tilde{\theta}} \rightarrow \mathbb{R}_{\geq 0}$, defined by $f(x):=$ $\max \left\{\sum_{i=1}^{N-2} x_{i}, \sum_{i=1}^{N-2}\left(\delta-x_{i}\right)\right\}$. Define the functions

$$
a(x):=\sum_{i=1}^{N-2} x_{i} \quad \text { and } \quad b(x):=\sum_{i=1}^{N-2}\left(\delta-x_{i}\right)
$$

and the sets

$$
\begin{aligned}
& A:=\left\{x \in I_{\tilde{\theta}}: a(x)>b(x)\right\} \\
& B:=\left\{x \in I_{\tilde{\theta}}: b(x)>a(x)\right\} \\
& C:=\left\{x \in I_{\tilde{\theta}}: a(x)=b(x)\right\}
\end{aligned}
$$

It then results that $I_{\tilde{\theta}}=A \cup B \cup C$, with $A \cap B=B \cap C=$ $C \cap A=\emptyset$. Observe, moreover, that we obviously have $\left.f\right|_{A}=\left.a\right|_{A}$ and $\left.f\right|_{B}=\left.b\right|_{B}$. By the fact that, for all $x \in C$, $\sum_{i=1}^{N-2} x_{i}=\sum_{i=1}^{N-2}\left(\delta-x_{i}\right)$, we have

$$
f(x)=a(x)=b(x)=\frac{N-2}{2} \delta, \quad \forall x \in C .
$$

Moreover, since $b(x)=(N-2) \delta-a(x)$, it results that

$$
a(x)>\frac{N-2}{2} \delta, \quad \forall x \in A,
$$

and

$$
b(x)>\frac{N-2}{2} \delta, \quad \forall x \in B .
$$

That is, since $\left.f\right|_{A}=\left.a\right|_{A},\left.f\right|_{B}=\left.b\right|_{B}$, and $A \cup B=I_{\tilde{\theta}} \backslash C$,

$$
f(x)>\frac{N-2}{2} \delta, \quad \forall x \in I_{\tilde{\theta}} \backslash C .
$$

From (83), this also implies that, for all $x \in I_{\tilde{\theta}}$,

$$
f(x) \geq \frac{N-2}{2} \delta
$$

From (81), (82), and (84), we obtain

$$
|\chi(\tilde{\theta})|_{2} \geq \frac{2}{\pi}\left(\delta+\frac{N-2}{2} \delta\right)=\frac{N}{\pi} \delta .
$$

Finally, recalling that $\delta=|\tilde{\theta}|_{\infty}$, by the norm inequality $|\tilde{\theta}|_{\infty} \geq \frac{|\tilde{\theta}|_{2}}{N-1}$, we conclude that, for all $\tilde{\theta} \in \mathcal{D}_{0}$,

$$
|\chi(\tilde{\theta})|_{2} \geq \frac{N}{\pi(N-1)}|\tilde{\theta}|_{2} \geq \frac{|\tilde{\theta}|_{2}}{\pi},
$$

which proves the claim.

\subsection{Proof of Claim 5}

Since we want to prove that $|\tilde{\theta}|_{2} \leq \sqrt{N} \delta \Rightarrow|\tilde{\theta}|_{\infty} \leq \delta$, we are going to prove that $|\tilde{\theta}|_{\infty} \geq \delta \Rightarrow|\tilde{\theta}|_{2} \geq \sqrt{N} \bar{\delta}$. It is enough to show that, for all $\delta \in[0, \pi]$,

$$
|\tilde{\theta}|_{\infty}=\delta \Rightarrow|\tilde{\theta}|_{2} \geq \sqrt{N} \delta
$$

Indeed, given $\delta^{\prime} \geq \delta$, (85) implies that, if $|\tilde{\theta}|_{\infty}=\delta^{\prime} \geq \delta$, then $|\tilde{\theta}|_{2} \geq \sqrt{N} \delta^{\prime} \geq \sqrt{N} \delta$. In order to prove (85) we minimize the Euclidean norm $|\tilde{\theta}|_{2}$ (or, equivalently, $|\tilde{\theta}|_{2}^{2}$ ), with the constraint that $|\tilde{\theta}|_{\infty}=\delta$. For the sake of simplicity, renumber the oscillator phases indexes in such a way that $\theta_{i} \leq \theta_{j}$ whenever $i<j$, as in the proof of Claim 3. The problem can then be translated into minimizing $|\tilde{\theta}|_{2}^{2}$, with the constraint that $\theta_{N}-\theta_{1}=\delta$. Since the square of the Euclidean norm and the constrained function are smooth, we can apply the method of Lagrange multipliers (see Appendix). That is, we can find critical points of $|\tilde{\theta}|_{2}^{2}$, under the constraint $\theta_{N}-\theta_{1}=\delta$, by solving the set of equations

$$
\begin{aligned}
& \frac{\partial}{\partial \theta_{i}} F(\theta, \lambda)=0, i=1, \ldots, N, \\
& \frac{\partial}{\partial \lambda} F(\theta, \lambda)=0,
\end{aligned}
$$

where $F(\theta, \lambda):=\sum_{i, j=1}^{N}\left(\theta_{i}-\theta_{j}\right)^{2}-\lambda\left(\theta_{N}-\theta_{1}-\delta\right)$, and $\lambda \in \mathbb{R}$ is the Lagrange multiplier. (86) gives, for $i \notin\{1, N\}$, gives

$$
\sum_{j=1}^{N}\left(\theta_{i}-\theta_{j}\right)=0
$$

While (87) gives the constraint $\theta_{N}-\theta_{1}=\delta$. In addition, (86) gives, for $i=1,4 \sum_{j=1}^{N}\left(\theta_{1}-\theta_{j}\right)+\lambda=0$, and, for 
$i=N, 4 \sum_{j=1}^{N}\left(\theta_{N}-\theta_{j}\right)-\lambda=0,$. By solving with respect to $\lambda$, we get

$$
\sum_{j=1}^{N}\left(\theta_{1}-\theta_{j}\right)+\sum_{j=1}^{N}\left(\theta_{N}-\theta_{j}\right)=0
$$

Equations (88)-(89), with the constraint $\theta_{N}-\theta_{1}=\delta$, admit a unique solution, modulo a common phase shift among the ensemble (i.e $\theta_{i} \rightarrow \theta_{i}+\alpha$ for all $i$ ):

$\theta_{i}^{*}-\theta_{j}^{*}=0, \quad \forall(i, j) \notin\{(1, N),(N, 1)\}$,
$\theta_{i}^{*}-\theta_{1}^{*}=\frac{\delta}{2}, \theta_{i}^{*}-\theta_{N}^{*}=-\frac{\delta}{2}, \forall i \notin\{1, N\}$.

By computing the Hessian of $F$ with respect to the vector $\left(\tilde{\theta}^{T}, \lambda\right)^{T}$, it is easy to show that its symmetric part is positive semidefinite for all $\left(\tilde{\theta}^{T}, \lambda\right)^{T}$. Hence the solution (90) corresponds to a minimum. To show the uniqueness of this critical point, modulo a common phase shift, note that the set of equations (86) can be rewritten as the linear system

$$
\begin{gathered}
\left(\begin{array}{ccccc}
N-1 & -1 & \ldots & -1 & -1 \\
-1 & N-1 & \ldots & -1 & -1 \\
\vdots & \vdots & \ddots & \vdots & \vdots \\
-1 & -1 & \ldots & N-1 & -1 \\
-1 & -1 & \ldots & -1 & N-1 \\
-1 & 0 & \ldots & 0 & 1
\end{array}\right)\left(\begin{array}{c}
\theta_{1} \\
\theta_{2} \\
\vdots \\
\theta_{N-2} \\
\theta_{N-1} \\
\theta_{N}
\end{array}\right) \\
= \\
\quad G \theta=\left(\begin{array}{c}
\frac{\lambda}{4} \\
0 \\
\vdots \\
0 \\
-\frac{\lambda}{4} \\
\delta
\end{array}\right)
\end{gathered}
$$

The matrix $G \in \mathbb{R}^{(N+1) \times N}$ has rank $N-1$, since the minor given by the first $N$ rows is the Laplacian matrix associated to a complete graph, which has rank $N-1$. In particular, it holds that $G \mathbf{1}_{N}=0$. Hence the solution to (91) is of the form $\theta^{*}=\theta_{\perp}^{*}+\alpha \mathbf{1}_{N}, \alpha \in \mathbb{R}$, where $\theta_{\perp}^{*}$ belongs to the ortogonal space to $\mathbf{1}_{N}$, and is uniquely determined by (91). This confirms that the solution (90) is unique, modulo a common phase shift. We can then conclude that, if $|\tilde{\theta}|_{\infty}=\delta$, then

$$
\begin{aligned}
|\tilde{\theta}|_{2}^{2} & \geq \sum_{i, j=1}^{N}\left(\theta_{i}^{*}-\theta_{j}^{*}\right)^{2} \\
& \geq 2 \delta^{2}+2 \sum_{j=2}^{N-2} \frac{\delta^{2}}{4}+2 \sum_{j=2}^{N-2} \frac{\delta^{2}}{4} \\
& \geq N \delta^{2}
\end{aligned}
$$

which proves the claim.

\section{A Lagrange Multipliers}

An extremum of a continuously differentiable function $f: \mathbb{R}^{n} \rightarrow \mathbb{R}$, under the constraints $g_{i}(x)=b_{i}, i=$ $1, \ldots, m$, where $g_{i}: \mathbb{R}^{n} \rightarrow \mathbb{R}$ is continuously differentiable, and $b_{i} \in \mathbb{R}$ belongs to the image of $g_{i}$, for all $i=1, \ldots, m$, can be found by constructing the Lagrangian function $F$ through the Lagrangian multipliers $\lambda_{i}, i=1, \ldots, m$,

$$
F\left(x, \lambda_{1}, \ldots, \lambda_{m}\right)=f(x)-\sum_{i=1}^{m} \lambda_{i}\left(g_{i}(x)-b_{i}\right)
$$

and by solving the set of equations

$$
\begin{gathered}
\frac{\partial}{\partial x_{i}} F\left(x, \lambda_{1}, \ldots, \lambda_{m}\right)=0, \\
\frac{\partial}{\partial \lambda_{j}} F\left(x, \lambda_{1}, \ldots, \lambda_{m}\right)=0,
\end{gathered}
$$

for all $i=1, \ldots, n$ and all $j=1, \ldots, m$. The optimal value $x^{*}$, is found together with the vector of Lagrangian multipliers $\lambda^{*}=\left(\lambda_{1}^{*}, \ldots, \lambda_{m}^{*}\right)$. See for example (Bliss, 1947).

\section{References}

Acebrón, J. A., Bonilla, L. L., Vicente, C. J. P., Ritort, F., Spigler, R., 2005. The Kuramoto model: A simple paradigm for synchronization phenomena. Reviews of modern physics 77, 137-185.

Aeyels, D., Rogge, J. A., 2004. Existence of partial entrainment and stability of phase locking behavior of coupled oscillators. Progress of Theoretical Physics 112 (6), 921-942.

Benabid, A. L., Pollak, P., Gervason, C., Hoffmann, D., Gao, D. M., Hommel, M., Perret, J. E., de Rougemont, J., 1991. Long-term suppression of tremor by chronic stimulation of the ventral intermediate thalamic nucleus. The Lancet 337, 403-406.

Billingsley, P., 1995. Probability and Measure, 3rd Edition. Probability and Mathematical Statistics. John Wiley and Sons, Inc., New York, NY. 
Bliss, G., 1947. Lectures on the calculus of variations. Chicago Univ. Press.

Brown, E., Holmes, P., Moehlis, J., 2003. Globally coupled oscillator networks. In: Sreenivasan, K., Kaplan, E., Marsden, J. (Eds.), Perspectives and Problems in Nonlinear Science: A Celebratory Volume in Honor of Larry Sirovich. New York.

Chopra, N., Spong, M. W., 2009. On exponential synchronization of Kuramoto oscillators. IEEE Trans. on Automat. Contr. 54 (2), 353-357.

Cumin, D., Unsworth, C., 2007. Generalising the Kuramoto model for the study of neuronal synchronisation in the brain. Physica D 226 (2), 181-196.

Daniels, B., 2005. Synchronization of globally coupled nonlinear oscillators: the rich behavior of the $\mathrm{Ku}$ ramoto model. Ohio Wesleyan Physics Dept., Essay, 7-20.Available on-line.

Dörfler, F., Bullo, F., 2011. Synchronization and transient stability in power networks and non-uniform Kuramoto oscillators. IEEE Trans. on Automat. Contr.(submitted).

Fradkov, A. L., 2007. Cybernetical Physics. From Control of Chaos to Quantum Control. Springer: Complexity. Springer-Verlag, Berlin Heidelberg.

Franci, A., Chaillet, A., Bezzaoucha, S., 2011. Toward oscillations inhibition by mean-field feedback in kuramoto oscillators. In: Proc. 18th. IFAC World Congress. To appear.

Franci, A., Chaillet, A., Pasillas-Lépine, W., 2010. Existence and robustness of phase-locking in coupled $\mathrm{Ku}-$ ramoto oscillators under mean-field feedback.

Hammond, C., Ammari, R., Bioulac, B., Garcia, L., 2008. Latest view on the mechanism of action of deep brain stimulation. Movement Disorders 23 (15), 21112121.

Hodgkin, A., Huxley, A., 1952. A quantitative description of membrane current and its application to conduction and excitation in nerve. J. Physiol 117, 500544.

Hoppensteadt, F. C., Izhikevich, E. M., 1997. Weakly connected neural networks. Vol. 126 of Applied Mathematical Sciences. Springer-Verlag, New York.

Isidori, A., 1999. Nonlinear control systems II. Springer Verlag, London, England .

Izhikevich, E. M., 2007. Dynamical Systems in Neuroscience: The Geometry of Excitability and Bursting. MIT Press, Cambridge, Massachusetts, London, England.

Jadbabaie, A., Motee, N., Barahona, M., 2004. On the stability of the Kuramoto model of coupled nonlinear oscillators. Proc. American Control Conf., 4296-4301.

Khalil, H., 2001. Nonlinear systems. Prentice Hall, 3rd ed., New York.

Krantz, S., Parks, H., 2002. A primer of real analytic functions. Birkhäuser, Boston - Basel - Berlin.

Kumar, R., Lozano, A. M., Sime, E., Lang, A. E., 2003. Long-term follow-up of thalamic deep brain stimulation for essential and Parkinsonian tremor. Neurology 61, 1601-1604.
Kuramoto, Y., 1984. Chemical Oscillations, Waves, and Turbulence. Springer, Berlin.

Lee, J., 2006. Introduction to smooth manifolds. Graduate Texts in Mathematics. Springer-Verlag, Berlin, Germany.

Lopez-Azcarate, J., Tainta, M., Rodriguez-Oroz, M. C., Valencia, M., Gonzalez, R., Guridi, J., Iriarte, J., Obeso, J. A., Artieda, J., Alegre, M., 2010. Coupling between beta and high-frequency activity in the human subthalamic nucleus may be a pathophysiological mechanism in Parkinsons disease. J. Neurosci. 30 (19), 6667-6677.

Loría, A., Panteley, E., 2005. Cascade nonlinear timevarying systems: analysis and design. In: LamnabhiLagarrigue, F., Loría, A., Panteley, E. (Eds.), Advanced topics in control systems theory. Lecture Notes in Control and Information Sciences. Springer Verlag.

Maistrenko, Y. L., Popovych, O. V., Tass, P. A., 2005. Desynchronization and chaos in the Kuramoto model. Lect. Notes Phys. 671, 285-306.

Malkin, I. J., 1958. Theory of stability of motion. Tech. rep., U.S. Atomic energy commission.

Nini, A., Feingold, A., Slovin, H., Bergman, H., 1995. Neurons in the globus pallidus do not show correlated activity in the normal monkey, but phase-locked oscillations appear in the MPTP model of Parkinsonism. J Neurophysiol. 74 (4), 1800-1805.

Olfati-Saber, R., Murray, R. M., 2004. Consensus problems in networks of agents with switching topology and time-delays. IEEE Trans. on Automat. Contr. 49 (9), 1520-1533.

Pavlov, A., van de Wouw, N., Nijmeijer, H., 2006. Uniform Output Regulation of Nonlinear Systems: a Convergent Dynamics Approach. Systems and Controls: Foundations and Apllications. Birkhauser, Boston.

Plenz, D., Kital, S. T., 1999. A basal ganglia pacemaker formed by the subthalamic nucleus and external globus pallidus. Nature 400 (6745), 677-682.

Popovych, O. V., Hauptmann, C., Tass, P. A., 2006. Desynchronization and decoupling of interacting oscillators by nonlinear delayed feedback. Internat. J. Bifur. Chaos 16 (7), 1977-1987.

Pyragas, K., Popovich, O. V., Tass, P. A., 2008. Controlling synchrony in oscillatory networks with a separate stimulation-registration setup. EPL 80 (4).

Rodriguez-Oroz, M. C., et al., 2005. Bilateral deep brain stimulation in Parkinson's disease: a multicentre study with 4 years follow-up. Brain 128, 2240-2249.

Rosa, M., Marceglia, S., Servello, D., Foffani, G., Rossi, L., Sassi, M., Mrakic-Sposta, S., Zangaglia, R., C, C. P., Porta, M., Priori, A., 2010. Time dependent subthalamic local field potential changes after DBS surgery in Parkinson's disease. Experimental Neurology $222(2), 184-190$.

Rosenblum, M., Pikovsky, A., 2004. Delayed feedback control of collective synchrony: an approach to suppression of pathological brain rhythms. Phys. Rev. E 70 (4), 041904.

Sarlette, A., 2009. Geometry and symmetries in coordi- 
nation control. Ph.D. thesis, University of Liège, (B).

Sarma, S. V., Cheng, M., Williams, Z., Hu, R., Eskandar, E., Brown, E. N., 2010. Comparing healthy and Parkinsonian neuronal activity in sub-thalamic nucleus using point process models. IEEE Trans Biomed Eng. 57 (6), 1297-1305.

Scardovi, L., Sarlette, A., Sepulchre, R., 2007. Synchronization and balancing on the N-torus. Syst. \& Contr. Letters 56 (5), 335-341.

Sepulchre, R., Paley, D., Leonard, N. E., 2008. Stabilization of planar collective motion with limited communication. IEEE Trans. on Automat. Contr. 53 (3), 706-719.

Sepulchre, R., Paley, D. A., Leonard, N. E., May 2007. Stabilization of planar collective motion: All-to-all communication. IEEE Trans. on Automat. Contr. $52(5), 811-824$.

Sontag, E. D., Wang, Y., 1996. New characterizations of Input-to-State Stability. IEEE Trans. on Automat. Contr. 41, 1283-1294.

Tass, P. A., 2003. A model of desynchronizing deep brain stimulation with a demand-controlled coordinated reset of neural subpopulations. Biol. Cybern. 89, 81-88.

Tukhlina, N., Rosenblum, M., Pikovsky, A., Kurths, J., 2007. Feedback suppression of neural synchrony by vanishing stimulation. Physical Review E 75 (1), 011918.

Van Hemmen, J. L., Wreszinski, W. F., 1993. Lyapunov function for the Kuramoto model on nonlinearly coupled oscillators. Jour. of Statistical Physics 72, 145166.

Volkmann, J., Joliot, M., Mogilner, A., Ioannides, A. A., Lado, F., Fazzini, E., Ribary, U., Llinás, R., 1996. Central motor loop oscillations in Parkinsonian resting tremor revealed by magnetoencephalography. Neurology 46, 1359-1370.

Winfree, A. T., 1980. The Geometry of Biological Times. Springer, New-York. 